\title{
What Does Visual Agnosia Tell Us About Perceptual Organization and Its Relationship to Object Perception?
}

\author{
Marlene Behrmann \\ Carnegie Mellon University
}

\author{
Ruth Kimchi \\ University of Haifa
}

\begin{abstract}
The authors studied 2 patients, S.M. and R.N., to examine perceptual organization and its relationship to object recognition. Both patients had normal, low-level vision and performed simple grouping operations normally but were unable to apprehend a multielement stimulus as a whole. R.N. failed to derive global structure even under optimal stimulus conditions, was less sensitive to grouping by closure, and was more impaired in object recognition than S.M. These findings suggest that perceptual organization involves a multiplicity of processes, some of which are simpler and are instantiated in lower order areas of visual cortex (e.g., collinearity). Other processes are more complex and rely on higher order visual areas (e.g., closure and shape formation). The failure to exploit these latter configural processes adversely affects object recognition.
\end{abstract}

The consciously perceived visual world is very different from the raw visual information or retinal mosaic of intensities and colors that arises from external objects. From the chaotic juxtaposition of different colors and shapes that stimulate the individual retinal receptors, an object is seen as detached and separable from adjacent objects and surfaces. This segmentation occurs despite the fact that parts of a single object may be spatially or temporally discontinuous, have different colors, or even transect several different depth planes. In addition, because most surfaces are opaque, portions of objects are routinely hidden from view and, as one moves around, surfaces continually undergo occlusion and fragmentation. As is apparent from this description, the objects of phenomenal perception are not given in any direct way in the retinal image. Some internal processes of organization must clearly be responsible, then, for producing a single, coherent percept. Exactly what these processes are remains poorly understood despite the roughly 100 years since the Gestalt psychologists first articulated the principles of perceptual organization. Although the

Authorship on this article is assigned alphabetically, and the work reflects an equal contribution by both authors. This research was supported by Grant MH47566 from the National Institute of Mental Health to Marlene Behrmann, by a United States-Israel Binational Science Foundation grant to both authors, and by the Weston Visiting Professorship at the Weizmann Institute of Science (Israel) to Marlene Behrmann. Some of the research was conducted while Ruth Kimchi was visiting Carnegie Mellon University and while Marlene Behrmann was visiting the Weizmann Institute.

We thank Thomas McKeeff, who helped with the data collection and statistical analysis, and Ilona Kovács, who graciously provided the material for the contour interpolation experiment. We are also grateful to the patients for their good-hearted and continued involvement in these experiments.

Correspondence concerning this article should be addressed to Marlene Behrmann, Department of Psychology, Carnegie Mellon University, Pittsburgh, Pennsylvania 15213-3890, or to Ruth Kimchi, Department of Psychology, University of Haifa, Mount Carmel, Haifa 31905, Israel. E-mail: behrmann@cnbc.cmu.edu or rkimchi@research.haifa.ac.il
Gestalt work on perceptual organization has been widely accepted as identifying crucial phenomena of perception, there has been, until the last decade or so, relatively little theoretical and empirical emphasis on perceptual organization, with a few exceptions. And, to the extent that progress has been made, there still remain many open questions.

In this article, we address three of these open questions. The first concerns the multiplicity of processes involved in deriving structure from a visual image. There is general consensus now that perceptual organization is not a monolithic entity but, rather, that several different processes exist. What these processes are and how they differ from one another in terms of their time course, attentional demands, and contribution to the ultimate goal of perceptual organization remains to be specified. A second question concerns the relationship between the various processes of perceptual organization and object recognition, and a final question relates to the brain systems that underlie these various perceptual organization processes.

\section{Perceptual Organization: A Multiplicity of Processes}

The Gestalt psychologists suggested that perceptual organization is achieved by grouping elements together by virtue of certain properties that are present in the image. In the seminal work of Wertheimer (1923/1950) and in the follow-up by Koehler (1928), the different heuristics underlying grouping have been enumerated, and they are now commonly listed in textbooks on perception. These heuristics include grouping by proximity, by closure, by similarity, by good continuation, and by common fate. Recently, Palmer (2001; Palmer \& Rock, 1994) added two more heuristics: grouping by common region and grouping by connectedness.

Despite the cataloguing and the widespread acceptance of these different grouping principles, many, although not all, theories of visual perception have treated perceptual organization as a unitary phenomenon that operates at a single, early, preattentive stage, in a bottom-up fashion, to create units which then serve as candidate objects for later and more elaborated processing, including object recognition and identification (Marr, 1982; Neisser, 1967; Treis- 
man, 1982; Treisman, Kahneman, \& Burkell, 1983). Several recent studies, however, have challenged such a view. For example, some researchers have argued that grouping does not occur as early as has been widely assumed but instead operates after depth information has been extracted (Rock \& Brosgole, 1964), and after lightness constancy (Rock, Nijhawan, Palmer, \& Tudor, 1992) and perceptual completion (Palmer, Neff, \& Beck, 1996; Palmer, 2001) have been achieved. Recent studies have also suggested that grouping requires attention (Mack, Tang, Tuma, Kahn, \& Rock, 1992), though other findings demonstrated that grouping can occur under conditions of inattention, without participants' conscious awareness (Driver, Davis, Russell, Turatto, \& Freeman, 2001; Moore \& Egeth, 1997; Razpurker-Apfeld \& Kimchi, 2003).

There have also been a host of recent studies that have proposed that not all grouping principles are created equal in terms of their time course, attentional demands, and relative weight in perceptual organization. For example, Kurylo (1997) obtained evidence suggesting that grouping by proximity requires less time than grouping by good continuity, and Ben-Av and Sagi (1995) and Han, Humphreys, and Chen (1999a) have shown that grouping by proximity is achieved faster than grouping based on similarity of shape. The features or identity of the visual elements are presumably critical for grouping based on similar shape, whereas spatial position information suffices for grouping by proximity. Kimchi (2000) demonstrated that proximity facilitated grouping by closure or by collinearity, but proximity had less impact when both collinearity and closure were present in the stimulus, and Donnelly, Humphreys, and Riddoch (1991) showed that a combination of closure and collinearity facilitated visual search more than closure alone. Palmer and Rock (1994) argued for an even more basic grouping heuristic, grouping by uniform connectedness, which precedes all other forms of grouping. According to this principle, a connected region of uniform visual property (such as color, texture, and motion) is perceived initially as a single perceptual unit. The claim that uniform connectedness has privileged status has been challenged, and several recent studies have suggested that it may not be as powerful as was initially proposed (Han, Humphreys, \& Chen, 1999b; Kimchi, 1998, 2000).

In addition to noting that grouping involves various principles or heuristics that may differ from each other, it appears that grouping itself may not be a single process. The Gestalt psychologists previously suggested that organization involves two distinct processes that are overlooked by most students of perception: the process of unit formation that determines which elements belong together (and are segregated from other elements) and the process of shape formation that determines the shape of the grouped elements (Koffka, 1935). Following the Gestaltists, Rock (1986) also suggested that organization has two meanings: grouping in the sense of what goes with what that refers to unit formation and configuring that determines the appearance of the grouped elements as a whole based on the interrelationships of the elements. On this account, grouping or element clustering is necessary for shape formation or configuring, but it is not identical to it. Trick and Enns (1997) have recently provided some evidence for this distinction. They showed that for neurologically intact participants, the enumeration of hierarchical figures (made of local elements) was as easy as the enumeration of connected line configurations, but when the stimuli to be enumerated were presented among distractors, the former was more difficult than the latter. Grouping of the local elements presumably suffices for the first enumeration task but not for the second in which shape discrimination was relevant, indicating that the further operation of shape formation was required in the second case.

\section{Perceptual Organization and Object Recognition}

The different processes of organization may also differ in their relative importance for the recognition of different visual objects. That is, specific grouping processes may be necessary and sufficient for the recognition of certain objects but not for others. It is interesting, however, that there has been little concerted effort to differentiate between the relative contribution of the different processes to object recognition. For example, Donnelly et al. (1991) have suggested that closure and good continuation are both particularly important for the derivation of shape descriptions. One may also conjecture that the product of grouping (in the sense of element clustering) may suffice for some forms of recognition but not others. For example, grouping collinear elements into a contour may be necessary and sufficient for simple line drawings that can be recognized by their contours, or the grouping of dots into a row may suffice for the detection of the row. Yet these types of grouping may not be sufficient for the recognition of more complex objects for which apprehension of the interrelationships and the configuring of the grouped elements is necessary.

Although not directly germane to our current focus but of importance to theories of visual perception more generally, the view that perceptual organization must precede object recognition, espoused by the traditional theories of perception, is also being challenged. Several different studies have produced evidence showing that knowledge of specific object shapes has an effect on grouping and figure-ground segregation. These findings are not compatible with the traditional linear or serial view of perceptual organization and object recognition, and more dynamic and interactive accounts have been proposed instead (Kimchi \& Hadad, 2002; Peterson \& Gibson, 1994a, 1994b; Vecera \& O’Reilly, 1998, 2000).

\section{Neural Mechanisms Underlying Perceptual Organization}

In addition to trying to understand the functional processes involved in perceptual organization, there is also much work to be done to understand how these principles are neurally instantiated and what brain mechanisms might be involved. Considerable neurophysiological advances have revealed much about the specificities of neuronal responses in visual cortex including their orientation selectivity, ocular dominance, wavelength, and directional selectivity. However, it is not clear how the fragments represented by these local analyzers are assembled to provide a unified percept.

It is worth noting that the Gestaltists did attempt to address the issue of neural implementation and attributed the Gestalt processes to isomorphic brain processes. For example, Kohler (1920/1950) conjectured that electromagnetic fields were the substrate of the brain's operation of a physical gestalt system. Although innovative in its time, this view was incorrect and, in fact, was one of the factors that contributed to the ultimate demise of the Gestalt perspective.

Recently, many studies involving single neuron recording in nonhuman primates as well as functional imaging in normal humans have been conducted to explore questions of perceptual organization. For example, there is a host of research on the 
perception of illusory contours (e.g., ffytche \& Zeki, 1996; Mendola, Dale, Fischl, Liu, \& Tootell, 1999; von der Heydt \& Peterhans, 1989; von der Heydt, Peterhans, \& Baumgartner, 1984) and the relationship of local fragments to a larger form (e.g., Georgopoulos et al., 2001; Hasson, Hendler, Ben Bashat, \& Malach, 2001; Op de Beeck, Beatse, Wagemans, Sunaert, \& Van Hecke, 2000) as well as several studies on the neural systems involved specifically in global-local form processing (Fink et al., 1996, 1997; Sasaki et al., 2001). Despite this flurry of recent activity, there remains much to be done to understand the mechanisms whereby light intensities are translated into meaningful objects by the brain.

\section{Integrative Agnosia}

One possible approach to understanding both the psychological and neural mechanisms involved in perceptual organization, and the one adopted here, is to study the performance of individuals who are impaired at the processes of perceptual organization following brain damage. The logic of this neuropsychological approach is equivalent to backward engineering: As researchers, if we can understand the operation of the system once it is unraveled, we might obtain insights into how it functions under normal circumstances (Coltheart, 2002). In the present article, we explore the behavior of 2 individuals, S.M. and R.N., whose impairment provides an ideal testing ground for investigating processes involved in perceptual organization and their relationship to object perception. The patients have a neuropsychological impairment, referred to as visual object agnosia, in which they are unable to recognize even familiar common objects presented to them in the visual modality. This object recognition deficit cannot be attributed to a problem in labeling the stimulus per se nor to a loss of semantics; presented with the same object in a different modality, either haptically or auditorily, they have no problem in naming it or providing detailed and rich descriptions of it. Visual agnosia refers to a specific failure to access the meaning of objects from the visual modality (Farah, 1990; Humphreys \& Riddoch, 2001; Ratcliff \& Newcombe, 1982).

Visual agnosia covers a wide spectrum of deficits, including, at one end, patients who are unable to recover even primitive features from a display (e.g., patients who fail to search in parallel for a vertical line among horizontal lines) and, at the other end, patients who appear able to extract a reasonably intact percept but subsequently fail to assign meaning to it (Farah, 1990; Humphreys \& Riddoch, 2001). The patients we have chosen to study fall midway between these two extremes: Their agnosia does not arise from impaired low-level vision nor from the inability to assign meaning to relatively intact visual representations, but rather from problems in organizational processes. The term applied to this deficit in intermediate vision is integrative agnosia and was coined by Riddoch and Humphreys (1987) on the basis of their studies with a patient called H.J.A. H.J.A. was impaired at search tasks that require the binding of visual elements in a spatially parallel manner across a field containing multiple stimuli; for example, he was disproportionately slowed, relative to control participants, in detecting the presence of an inverted $T$ among upright $T \mathrm{~s}$. In contrast, his search is efficient and rapid for targets that do not require a combination of elements such as a target diagonal among multiple verticals (Humphreys, 1999; Humphreys \& Riddoch, 1987; Humphreys et al., 1994; Humphreys, Riddoch, Quinlan, Price, \& Donnelly, 1992). When the demands for integration are low, H.J.A. and other integrative agnosic patients perform significantly above chance levels: They can make same-different judgments accurately on two stimuli that share area and brightness but not shape (aspect ratio changes from square to rectangle; Efron, 1968).

In addition to the impaired ability to integrate all aspects of the display into a whole, several other characteristics now serve as the core features of integrative agnosia (Behrmann, in press; Behrmann \& Kimchi, in press; Humphreys \& Riddoch, 2001). For example, the patients are more impaired at identifying items that overlap one another compared with the same items presented in isolation. It is interesting and also counterintuitive that, in some patients, the presence of local information may even reduce the efficiency of visual recognition; in contrast with normal perceivers, both patients H.J.A. (Lawson \& Humphreys, 1999; Riddoch \& Humphreys, 1987) and S.M. (Butter \& Trobe, 1994) identified silhouettes better than line drawings whose internal details apparently led to incorrect segmentation. Patients with integrative agnosia also fail to identify shapes by subjective contours and do not appreciate occlusion normally. Another key feature of the disorder is the failure to segregate figures from ground effectively; for example, patient F.G.P. was unable to detect a simple shape against a pattern background (Kartsounis \& Warrington, 1991). Finally and critically for our purposes, integrative agnosic patients are impaired at grouping elements of a display. A clear example comes from patient N.M., who was impaired at detecting the presence of a target letter that was formed by grouping local elements that differed from the background element by line orientation (texture), color, luminance, or motion (Ricci, Vaishnavi, \& Chatterjee, 1999; see also, Kartsounis \& Warrington, 1991; Marstrand, Gerlach, Udesen, \& Gade, 2000). Given the paucity of research on integrative agnosia, a definitive definition is not yet established, but the critical component is a failure in organizational processes in tandem with a deficit in object recognition.

Patients with integrative agnosia are also obviously impaired at object recognition: As is evident from their responses to black and white line drawings (see Figure 1), the 2 patients we studied are clearly able to extract some visual information from the display but apparently are unable to integrate all aspects into a meaningful whole. The problem applies equally to the recognition of two- and three-dimensional stimuli and to black and white and chromatic displays although, in some cases, the presence of depth, color, and surface cues may be of some assistance to the patients in segmenting the display (Chainay \& Humphreys, 2001; Farah, 1990; Humphrey, Goodale, Jakobson, \& Servos, 1994; Jankowiak, Kinsbourne, Shalev, \& Bachman, 1992).

We start off by describing the 2 agnosic patients we have studied. At the outset, we had some indication that the 2 patients were differentially impaired in their object recognition ability, and we exploited this fact in investigating the nature of the grouping impairment and the way in which various grouping processes might differentially contribute to object recognition.

\section{Description of Cases}

Because the same patients participated in all the experiments, we describe them here at the outset. Control participants were standardly run in each experiment, but because the exact control group differed from one experiment to another, we describe the control participants separately for each experiment. 
A

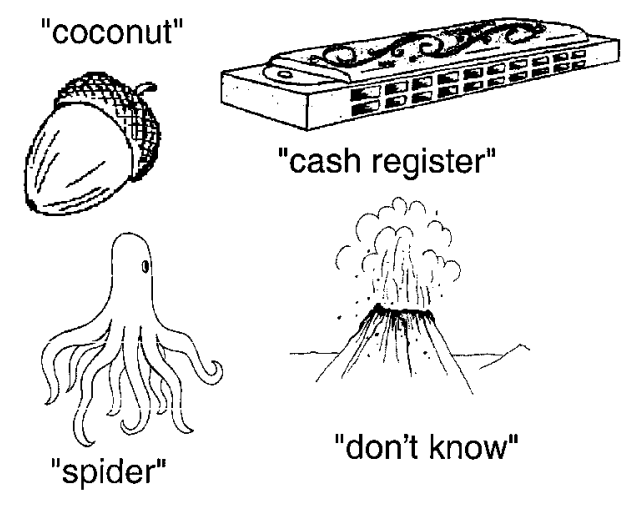

B

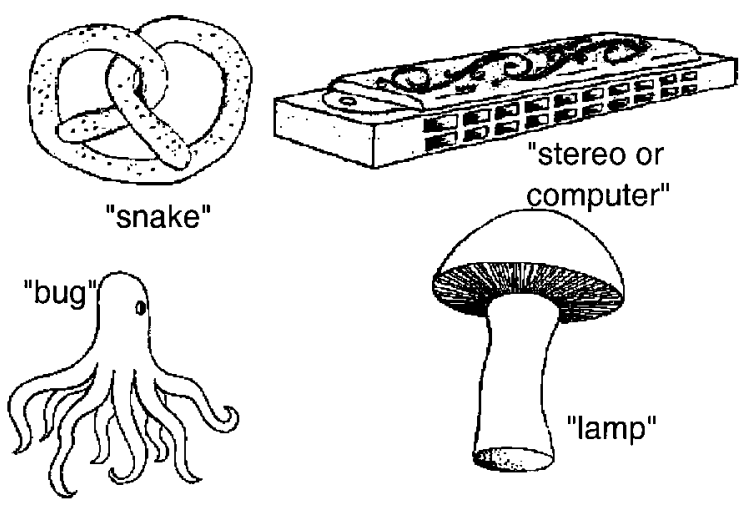

Figure 1. Examples of black and white line drawings and the responses of S.M. (A) and R.N. (B) to these pictures. From Boston Naming Test (2nd ed.; acorn, p. 32; harmonica, p. 30; mushroom, p. 14; pretzel, p. 19; octopus, p. 13; volcano, p. 23), by H. Goodglass, E. Kaplan, and S. Weintraub (O. Segal, Illus.), 1983, Philadelphia: Lea \& Febiger. Copyright 1983 by Lippincott Williams \& Wilkins. Reprinted with permission.

Two adult male patients, S.M. and R.N., both of whom are right-handed and English-speaking, consented to participate. Both had been diagnosed as having visual agnosia and had participated in several previous studies (Behrmann, in press; Behrmann \& Kimchi, in press; Gauthier, Behrmann, \& Tarr, 1999; Marotta, Behrmann, \& Genovese, 2001; Marotta, McKeeff, \& Behrmann, 2002). Because extensive information is available about them in these other publications, we present only a short review of their biographical and medical histories.

S.M. is a young man with visual acuity corrected to $20 / 20$. He sustained a closed head injury in a motor vehicle accident in 1994 at the age of 18 , and the experiments reported here were conducted in 1998 and 1999. Despite extensive injuries, he recovered extremely well and the only residual deficit is the visual agnosia. Figure 2 presents MRI images for S.M. demonstrating the site and extent of his inferior temporal lobe lesion (Marotta et al., 2001). Note that although S.M. is right-handed, he has some weakness on the right side as his arm was badly damaged in the accident, and so he uses his left hand intermittently (and responds in these experiments with his left hand). At the time of the accident, S.M. was about to begin college. After several years of rehabilitation, he has returned to his studies (although he requires considerable assistance with the visual material) and works in his family's photographic store.

R.N. experienced a myocardial infarction in 1999 at the age of 39 , and the experiments reported here were started 6 months thereafter. His visual acuity is $20 / 20$. He worked in his own construction business at the time of the infarction and is not employed at present. R.N. does not have any focal lesion on his MRI scan; the absence of a circumscribed lesion from a patient who has sustained brain damage following a myocardial infarction during bypass surgery is not uncommon. ${ }^{1}$ Because the neuropil is generally preserved after such an incident even if the neurons themselves are affected, a circumscribed lesion may not be detectable even with high-resolution imaging. ${ }^{2}$

Both patients performed normally on those subtests of the Birmingham Object Recognition Battery (BORB; Riddoch \& Humphreys, 1993) that tap low-level or early visual processes, including judging line length, orientation, size, and gap position. Both patients also had normal color vision. That both patients could derive considerable visual information is further supported by their performance on a copying task; both patients produced reasonably good copies of a target object or a scene (see Figure 3), although they did so slowly relative to normal participants and in a labored and segmental fashion. Both patients also performed within normal limits on more complex visual tasks, such as matching objects on the basis of minimal features or when one object was foreshortened. It is important to note, however, that both patients were impaired on the BORB subtests that evaluate discrimination of overlapping shapes, and both performed in the impaired range on the object decision subtests (task: Is this a real object or not?), as is usually the case with patients with integrative agnosia. In contrast with some integrative agnosic patients (Butter \& Trobe, 1994; Lawson \& Humphreys, 1999; Riddoch \& Humphreys, 1987), neither S.M. nor R.N. performed better with silhouettes than with line drawings. See Figure 4 for examples of the different types of stimuli.

Both patients read accurately but slowly as reflected in their response times to read-aloud words of different lengths presented individually on the computer screen. Whereas normal readers show minimal, if any, effect of word length on reading time within this range ( 3 to 8 letters in length), both patients had increased slopes relative to control participants. Whereas S.M. read 117/120 words correctly with a slope of 104 ms per additional letter, R.N. read 95/120 words correctly with a 241-ms slope (Mycroft, Behrmann, \& Kay, 2002). Both patients were also significantly impaired at face recognition (see Gauthier et al., 1999; Marotta et al., 2002).

Both performed normally in naming objects presented to them in the haptic modality, while blindfolded, or in the auditory modality, and both could provide rich definitions of the objects they

\footnotetext{
${ }^{1}$ We thank H. B. Coslett for discussing R.N.'s neurological status with us.

${ }^{2}$ We attempted a functional imaging scan on R.N., but he is too large to remain in the $3 \mathrm{~T}$ scanner for any length of time, and so these data could not be obtained.
} 

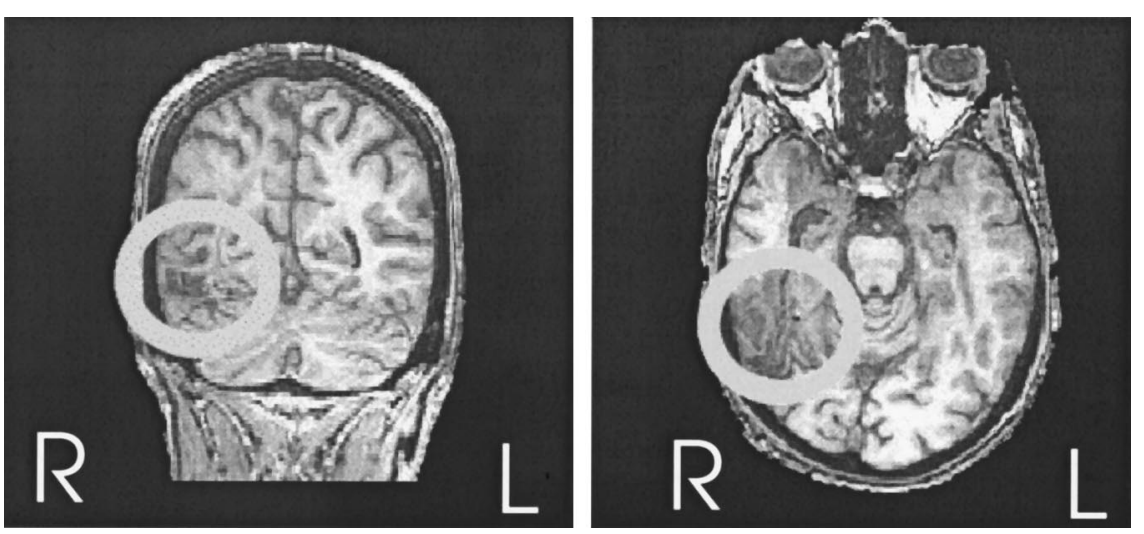

Figure 2. Structural scan from S.M. showing the localization of the lesion to the right inferior temporal lobe.

failed to recognize in the visual modality. The preserved naming performance and ability to define the objects ruled out both an anomia and a semantic deficit as the underlying cause of the object recognition failure. The patients also did not have available to them information about the display that they could indicate via gesture, as is the case in individuals suffering from optic aphasia. The deficit in these 2 patients is restricted to the inability to recognize images presented visually.

\section{Experiment 1: Object Recognition}

In this experiment, we document the patients' visual object recognition abilities.

\section{Method}

Participants. S.M. and R.N. participated in this experiment.

Stimuli and procedure. The 260 black and white line drawings from the Snodgrass and Vanderwart (1980) set were scanned into individual files

B

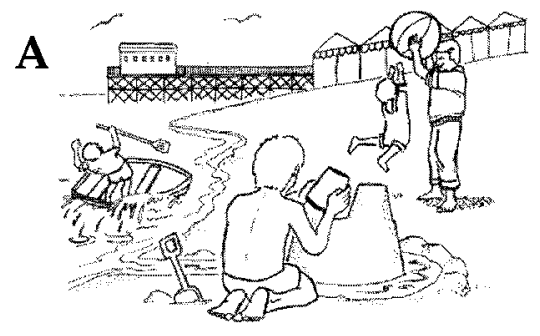

C
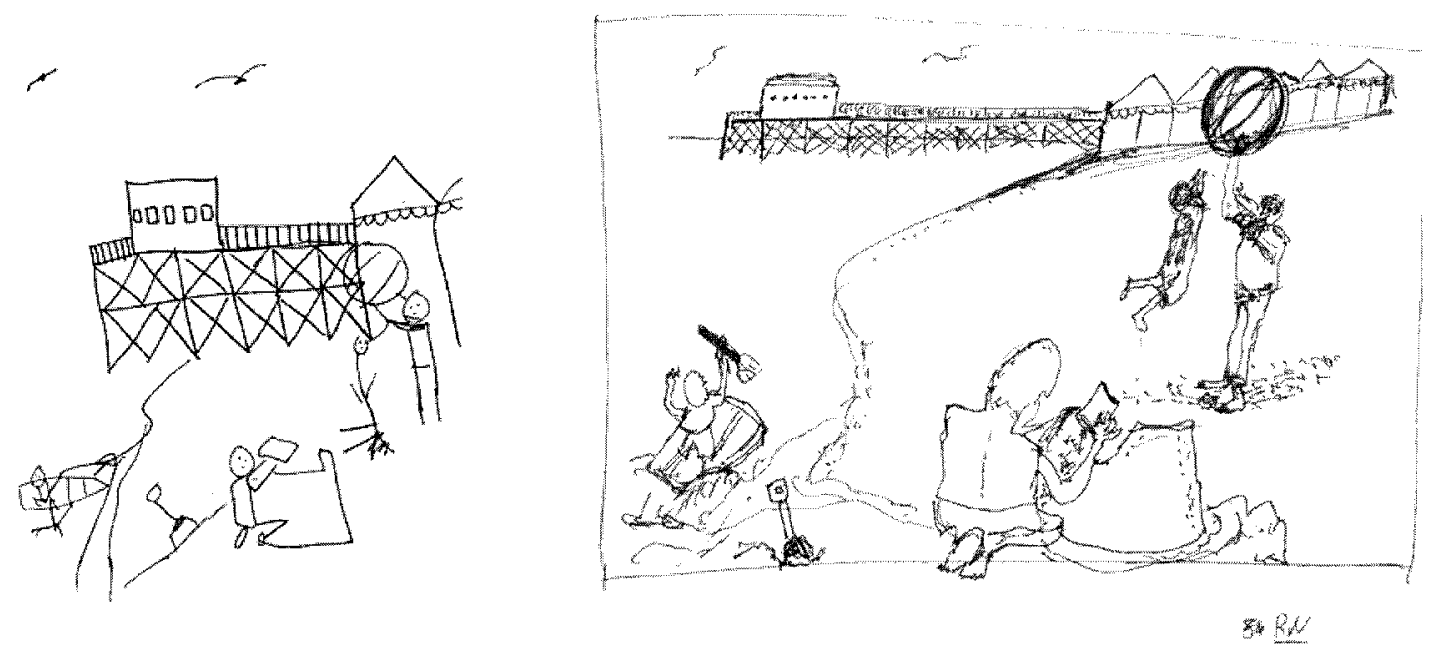

Figure 3. Display of a beach scene (A) with the copies by S.M. (B) and R.N. (C), both of whom took an inordinate amount of time to complete this task. 
A

B

\section{TJM}
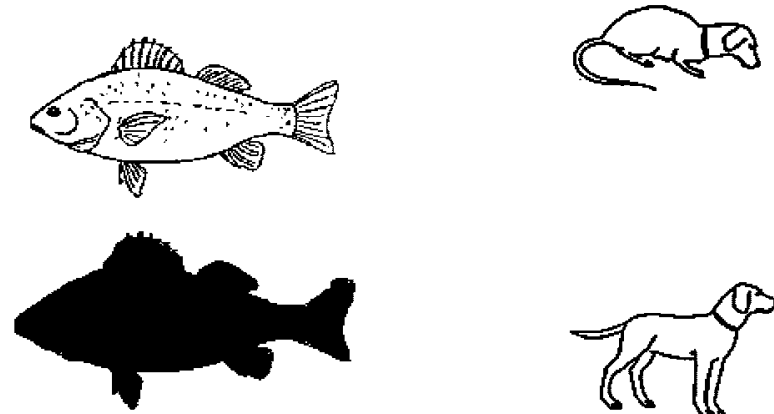

C

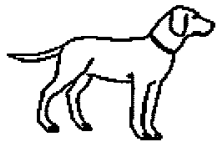

Figure 4. Examples of overlapping and individual letters (A), line drawings for object decision (B), and silhouettes for object identification (C). The line drawings in panel B are from "A Standardized Set of 260 Pictures: Norms for Name Agreement, Image Agreement, Familiarity, and Visual Complexity," by S. G. Snodgrass \& M. A. Vanderwart, 1980, Journal of Experimental Psychology: Human Learning and Memory, 6, pp. 199, 201. Copyright 1980 by the American Psychological Association. Adapted with permission of the authors.

and presented centrally on a Macintosh Quadra computer screen (19 in. or $48.26 \mathrm{~cm}$ ) for identification using PsyScope software (Cohen, MacWhinney, Flatt, \& Provost, 1993). The images remained exposed until response and both accuracy and vocal reaction time (RT) were measured. The experimenter recorded the label used by the patient. The displays ranged in size, with the largest and smallest subtending $9.0^{\circ} \times 10.8^{\circ}$ and $6.0^{\circ} \times 7.4^{\circ}$, vertically and horizontally, respectively. Participants were encouraged to respond both quickly and accurately here and in the experiments that follow in which both accuracy and RT are measured.

\section{Results and Discussion}

S.M. identified $66 \%(171 / 260)$ of the objects, whereas R.N. identified only $51 \%(132 / 160)$, reflecting a significant difference in their object recognition abilities, $\chi^{2}(1, N=159)=11.42, p<$ .001 . Neither appeared to be exhibiting a speed-accuracy trade-off as S.M. required an average of $2.14 \mathrm{~s}$ per image, whereas R.N. averaged $8.52 \mathrm{~ms}$ per image, confirming the greater impairment in R.N. than S.M., $F(1,350)=53.17, M S E=3,379,000,000, p<$ .0001 . In a group of nonneurological participants covering a wide age range (Behrmann, Nelson, \& Sekuler, 1998), accuracy was $96.4 \%$, and the mean RT was $884.67 \mathrm{~ms}$. Both patients showed accuracy and RTs more than three standard deviations from these means. The errors made by the patients were mostly visual confusions (see Table 1; also shown in Figure 1) and indicate that the patients were deriving considerable visual information from the display but appeared not to take into account all aspects of the display.

The findings from the object recognition task indicate first that both patients are significantly impaired in their ability to identify objects, relative to normal participants. Two aspects of the data are informative, however: The first is that the patients are inordinately slow in identifying objects, and this is consistent with the possibility that they build up their representations slowly and in a segmental fashion. The second is that R.N. is significantly impaired relative to S.M. in both accuracy and RT. These two findings are also apparent in their word reading; there is a linear increase in RT when naming a word as the number of letters increases (see above), and this is so to a greater extent for R.N. than for S.M. In sum, both S.M. and R.N. exhibit the characteristics of integrative agnosia. However, the deficit in object recognition is more severe in the case of R.N. than in S.M.

\section{Experiment 2: Perception of Global Configuration in Hierarchical Stimuli}

We now explore the patients' abilities to integrate aspects of a display into a coherent configuration and then evaluate how this is related to their object recognition impairment. In this experiment, we examined whether the patients could perceive the global configuration of a pattern made of elements. This is often considered to be a measure of grouping and element integration (Enns \& Kingstone, 1995; Han \& Humphreys, 1999; Han et al., 1999a). This experiment uses a now-standard stimulus, the Navon-type hierarchical stimulus in which a global letter is made up of local letters having either the same or different identity as the global letter (see Figure 5). Participants typically identify the letter at either the global or local level in separate blocks of trials (in the focused attention version of the task). All else being equal, the global letter is identified faster than the local letter, and conflicting information between the global and the local levels exerts asymmetrical global-to-local interference (Navon, 1977). Although the mechanisms underlying this global advantage are still disputed, the phenomenon is robust and is observed under various exposure durations, including short ones (e.g., Navon, 1977; Paquet \& Merikle, 1984; Yovel, Yovel, \& Levy, 2001), suggesting that normal participants can easily and quickly perceive the global configuration of hierarchical patterns (see Kimchi, 1992, for a review).

The obvious prediction one might make is that patients who experience difficulties in grouping the local elements would be impaired at deriving the global configuration and would therefore be slowed in detecting the global letter. In addition, the enhanced

Table 1

Object Recognition Errors Made by S.M. and R.N.

\begin{tabular}{|c|c|}
\hline Target & Response \\
\hline \multicolumn{2}{|c|}{ Patient S.M. } \\
\hline Coat & Shirt \\
\hline Fox & Dog \\
\hline Mountain & Tent \\
\hline Cheetah & Horse \\
\hline Nail file & Knife \\
\hline Monkey & Rat \\
\hline Cat & Hamster \\
\hline
\end{tabular}

Patient R.N

$\begin{array}{ll}\text { Tie } & \text { String } \\ \text { Fox } & \text { Dog } \\ \text { Mountain } & \text { Spider } \\ \text { Cheetah } & \text { Sheep } \\ \text { Salt shaker } & \text { Can } \\ \text { Toe } & \text { Finger } \\ \text { Nose } & \text { String } \\ \text { Bow } & \text { Bee } \\ \text { Lemon } & \text { Potato }\end{array}$




\section{CONSISTENT}

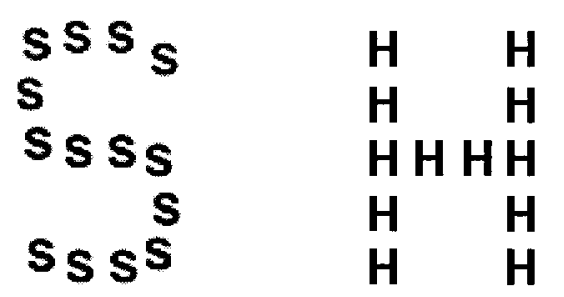

\section{INCONSISTENT}

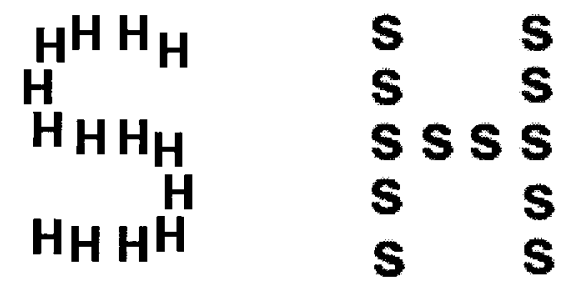

Figure 5. Hierarchical stimuli that are two letters, $H$ and $S$, composed of $H \mathrm{~s}$ or $\mathrm{Ss}$.

processing of the local elements might interfere with the derivation of the global identity: Thus, we might see interference when the participants identify the stimulus at the global level and there is inconsistency between the identity of the local and the global letter.

\section{Method}

Participants. The 2 patients, S.M. and R.N., and a group of nonneurological participants completed this experiment. The control participants, consisting of 10 university students (8 men and 2 women, mean age 18.7 years) drawn from the participant pool of the Department of Psychology, Carnegie Mellon University, volunteered to participate in return for course credit. Participants had normal or corrected-to-normal visual acuity by self-report, and all but 1 were right handed.

Apparatus and material. The experiment was conducted on a Macintosh Powerbook 540C (with a 9.5-in. or 24.13-cm monitor) or a Macintosh Quadra 650 (with a 15 -in. or $38.1 \mathrm{~cm}$ monitor) and was executed with PsyScope experimental software, Version 1.2.1 (Cohen, MacWhinney, Flatt, \& Provost, 1993). All responses were recorded on a Button Box (New Micros, Dallas, TX).

The stimuli were four hierarchical letters of two types: consistent letters, in which the global and the local letters shared identity (a large $H$ made of small $H \mathrm{~s}$ and a large $S$ made of small $S \mathrm{~s}$ ), and inconsistent letters in which the letters at the two levels had different identities (a large $H$ made of small $S$ s and a large $S$ made of small $\mathrm{Hs}$; see Figure 5). The global letter subtended $3.2^{\circ}$ in height and $2.3^{\circ}$ in width, and the local letter subtended $0.44^{\circ}$ in height and $0.53^{\circ}$ in width.

Design and procedure. Participants sat in a dimly lit room and used a chin rest to restrict head mobility and allow a fixed viewing distance of 65 $\mathrm{cm}$. The experiment consisted of the factorial combination of two variables in a repeated measures design: task (global vs. local identification) and consistency (consistent vs. inconsistent stimuli). The two tasks, local or global identification, were administered in separate blocks of 96 experi- mental trials each, preceded by 10 practice trials. The consistent and inconsistent letters were randomized within block, with each letter occurring on an equal number of trials. The normal participants completed 192 experimental trials, and the patients completed 384 experimental trials across two sessions. Before each block, participants were verbally instructed to respond to the global or local letters. Each trial was initiated with a central fixation cross of $500 \mathrm{~ms}$ duration. This was immediately replaced by one of the four possible stimuli, which remained on the screen until a response was made. Participants were instructed to press the right key on the button box to indicate a response of $S$ or the left key for $H$. The order of the blocks and response designation was counterbalanced across participants.

\section{Results and Discussion}

Mean correct RTs for the global and local identification are presented in Figure 6 as a function of stimulus consistency for the normal participants (A) and for each of the patients (B and C).

The data from the normal participants were subjected to a two-way analysis of variance (ANOVA) with globality (global, local) and consistency (consistent, inconsistent) as within-subject factors. The data from each individual patient were analyzed separately with the same two factors as between-subject factors and trial type as the random variable.

The normal participants were extremely accurate in identifying the letters, reporting $96.3 \%$ correctly. As reflected in Figure 6, they showed a small but significant global advantage of $15 \mathrm{~ms}, F(1$, $9)=4.5, p=.06, M S E=2,175$. There was no difference between consistent and inconsistent items, nor was there an interaction between globality and consistency, $F \mathrm{~s}<1$, although there was a numeric trend toward interference in local identification in the inconsistent case, suggesting some interference from the global identity onto the local identification. The absence of strong interference effects is not unusual given the unlimited exposure duration (Paquet \& Merikle, 1984), foveal presentation (Pomerantz, 1983), and spatial certainty (Lamb \& Robertson, 1988). What is more relevant for our purposes is how the patients performed relative to this control baseline.

Both patients were highly accurate, with S.M. and R.N. achieving $98.9 \%$ and $99.1 \%$ accuracy, respectively. S.M.'s pattern of performance was not that different from that of the normal participants. He showed a significant global advantage of $58 \mathrm{~ms}, F(1$, $377)=13, p<.0005$, but there was no consistency effect, nor was there an interaction between globality and consistency, $F_{\mathrm{s}}<1$, although, as in the normal participants, there was also a numeric trend for global-to-local interference.

R.N. exhibited a dramatically different pattern, showing a clear local advantage and identifying local letters $174 \mathrm{~ms}$ faster than global letters, $F(1,375)=55.7, p<.0001, M S E=2,914,963$. He was also $149 \mathrm{~ms}$ faster for consistent over inconsistent stimuli, $F(1,375)=12.4, p<.0005, M S E=648,276$, but this consistency effect was qualified by an interaction with globality, $F(1,375)=$ $10.4, p<.001, M S E=546,143$. The interaction reveals that although there was only a 7-ms difference between consistent and inconsistent trials in the local condition, there was a $159-\mathrm{ms}$ slowing for the inconsistent over consistent trials in the global condition, reflecting strong local-to-global interference.

The findings from the global-local task reveal a major discrepancy in the performance of the 2 patients. S.M. performed qualitatively similarly to normal participants: Responses were faster with global than with local stimuli, and there was a trend toward 

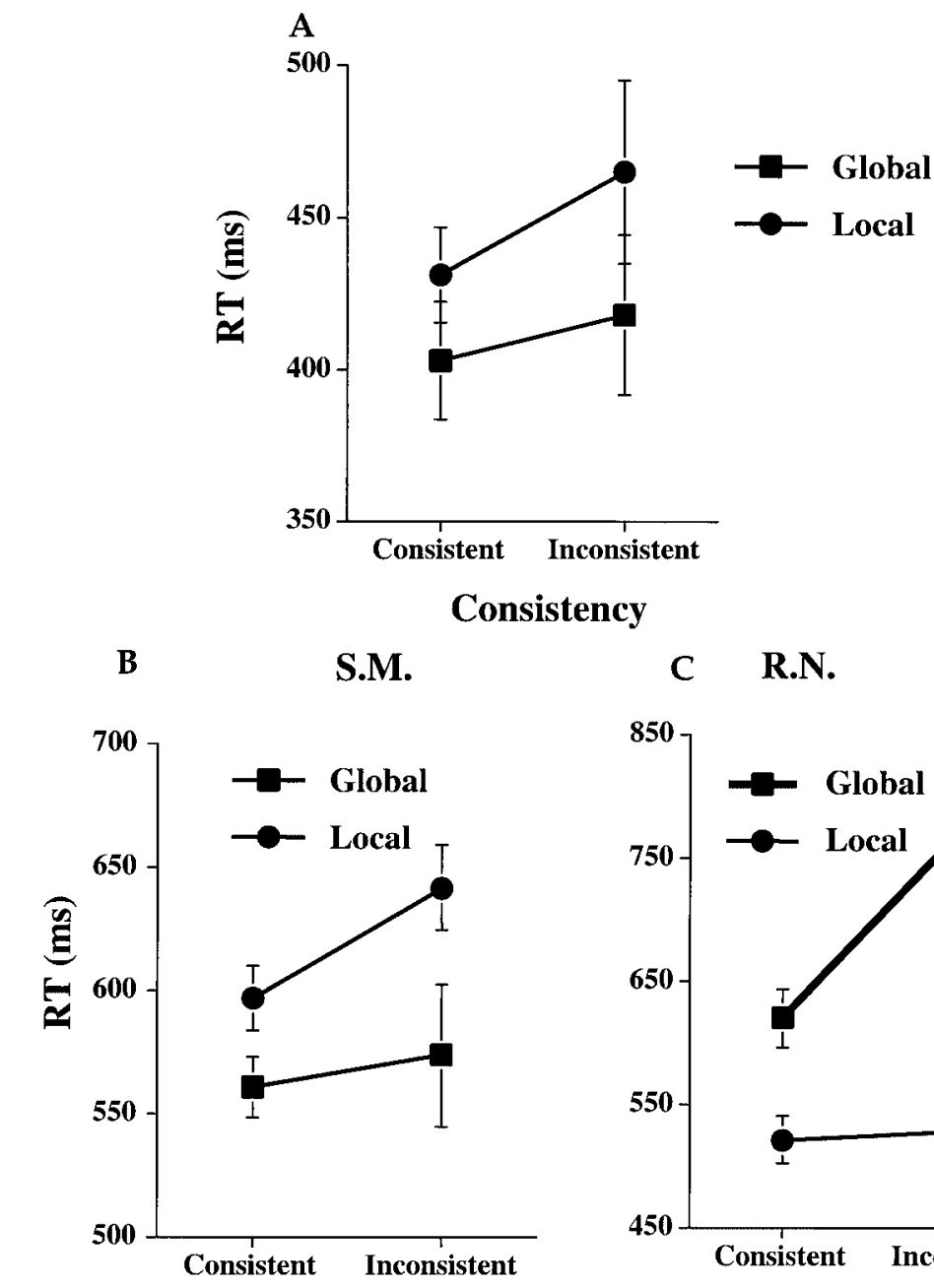

Consistency

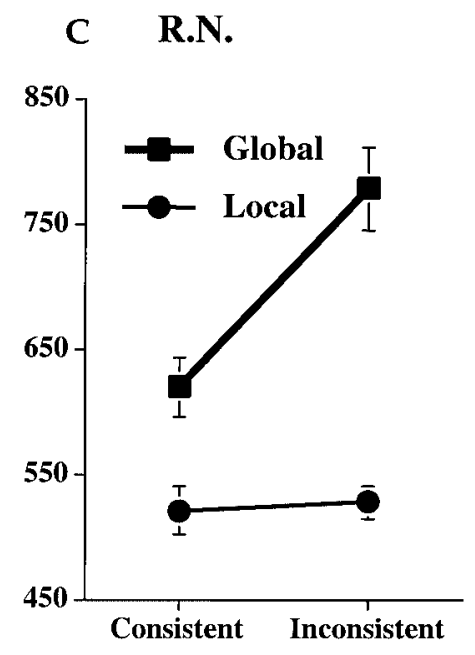

Consistency

Figure 6. Mean response times (RTs) for the control subjects (A), for S.M. (B), and for R.N. (C) to indicate letter identity as a function of consistency between the local and global levels. Note the different $y$-axes across the three graphs. Error bars represent standard errors of the means.

global-to-local interference. R.N., on the other hand, was faster with local than global letters and showed strong interference from the local letter onto global identification when there was inconsistency between the two.

A finer analysis of the data in which we looked at performance for the different letters ( $H$ vs. $S$ ) revealed another interesting difference between S.M. and R.N. When making global identifications, both patients responded faster to $H$ than to $S$. However, S.M. responded to the global $H$ made of $H$ s $(537 \mathrm{~ms})$ as fast as to the global $H$ made of $S \mathrm{~s}$ (544 ms). R.N., on the other hand, was 133 ms faster in responding to the global $H$ made of $H \mathrm{~s}(605 \mathrm{~ms})$ than to the global $H$ made of $S \mathrm{~s}$ (738 ms), and furthermore, the former was the only case in which his global identification was nearly as fast as his local identification of $H(565 \mathrm{~ms})$.

The discrepancy between the patients in their ability to apprehend the global configuration of patterns composed of elements presumably reflects different types of deficits in perceptual organization, or perhaps different levels of deficits. Assuming that the local elements of the hierarchical letters are grouped by proximity and/or similarity (the elements are identical and close to one another), R.N. seemed unable to use these grouping principles to derive the global configuration; he could derive some global structure only when collinearity between elements was present (as in the case of an $H$ made of $H$ s). S.M., on the other hand, appeared able to derive a global configuration even when simple collinearity was not present in the image. We pursue this issue further in later experiments.

It is interesting that a similar discrepancy between global-local performance exists between 2 other patients in the literature. H.J.A., perhaps the most extensively studied patient with integrative agnosia, showed an advantage for global over local identification but showed no interference of any kind (Humphreys, 1999; Humphreys \& Riddoch, 2001). In contrast, N.M., who is also a very good example of an integrative agnosic patient, was almost unable to identify the global letter even at unlimited exposure duration (Ricci et al., 1999) and favored reporting the local components. 
The variability observed across patients on this task suggests that a problem in deriving global structure might not be a core element of integrative agnosia. This conclusion might be premature, however. It is now well known that a variety of stimulus and task factors affect the balance between global and local processing, including the type of hierarchical stimuli used, the attentional task (divided or focused), and the mode of response (forced choice, go/no-go) (Kimchi, 1992; Yovel et al., 2001). Thus, the variability in the pattern of results obtained across patients might be a function of the different testing conditions used with different patients. We note that the experimental conditions used here are favorable for deriving a global configuration: focused attention (blocked by local vs. global response) and global stimulus saliency (in which many small elements increase the salience of the global over the local letters; e.g., Yovel et al., 2001). Under these conditions and with unlimited exposure duration, S.M. was able to derive the global configuration but, as we will see below, under more stringent testing conditions, even S.M. exhibited an impairment in global processing. These findings further support the claim that differences in testing conditions may lead to variability in outcome. Alternatively, because perceptual organization refers to a multiplicity of processes, it is possible that patients do vary and that integrative agnosia might manifest in different ways across different individuals. Because such individuals are rare, the opportunity to systematically analyze all their perceptual skills in depth is not that easy, and so the source of this cross-patient variability remains to be determined.

In the following experiment, we explore further the question of perceptual organization and global structure. Before we do so, however, we need to rule out the possibility that the differential ability of the 2 patients in deriving the global configuration is not simply due to a differential sensitivity to high and low spatial frequencies, which we do in the next experiment.

\section{Experiment 3: Spatial Frequency Thresholds}

Several researchers have suggested an involvement of spatial filters, based on spatial frequency channels, operating at early visual processing (Ginsburg, 1986) in the perception of global and local structures. For example, in a number of these studies, no latency advantage for global over local processing was found when low-spatial frequencies were removed from hierarchical stimuli (Badcock, Whitworth, Badcock, \& Lovegrove, 1990; Hughes, Fendrich, \& Reuter-Lorenz, 1990; Lamb \& Yund, 1993; Shulman, Sullivan, Gish, \& Sakoda, 1986; Shulman \& Wilson, 1987), suggesting that the global advantage effect is mediated by low-spatialfrequency channels. Thus, one possible explanation for the patients' differential inability to perceive the global form of a hierarchical stimulus might concern a fundamental limitation in processing low-spatial-frequency information. The obvious prediction from this in relation to the patients is that R.N., who appears to process stimuli almost entirely at the local level, should be impaired at processing low-frequency displays, resulting in an increased low-spatial-frequency threshold, relative to control participants, whereas S.M., who shows some global form processing, should not show as much of an increase in this threshold. In Experiment 3, we establish thresholds for both patients across a wide range of spatial frequencies and compare them to those of control participants.

\section{Method}

Participants. In addition to S.M. and R.N., 10 normal male participants, with a mean age of 27.7 years (range $=19$ to 51 years) participated in this experiment. Nine of the participants were right-handed, and all had normal or corrected-to-normal vision.

Apparatus and procedure. To document the spatial frequency function, we established for each individual the log contrast thresholds at 1,3 , 5, 10, and 30 cycles per image (CPI) using a MATLAB (Brainard, 1997) function that implements a discrimination task. Participants completed five blocks of trials, with each block containing 20 trials and using displays corresponding to one of the CPIs (examples of stimuli at 1 and 30 CPIs are shown in Figure 7). In each trial, a fixation point appeared on the screen for $1 \mathrm{~s}$. After $200 \mathrm{~ms}$, one image appeared for $200 \mathrm{~ms}$ followed by a blank screen for a further $200 \mathrm{~ms}$. A second image then appeared for $200 \mathrm{~ms}$ and it, in turn, was replaced by a $200-\mathrm{ms}$ blank screen. At this point, the participant was required to decide whether the first or second image contained the grating. Feedback was provided to the participant after each trial. A series of five practice trials was presented before the first block, and the order of blocks was counterbalanced across participants. If the response was correct, a more difficult discrimination (decreased contrast by 0.2 ) was presented on the next trial. If the response was incorrect, the contrast was increased by 0.2. A log contrast threshold was determined for each CPI using a method of limits in which threshold was defined as the value of
A

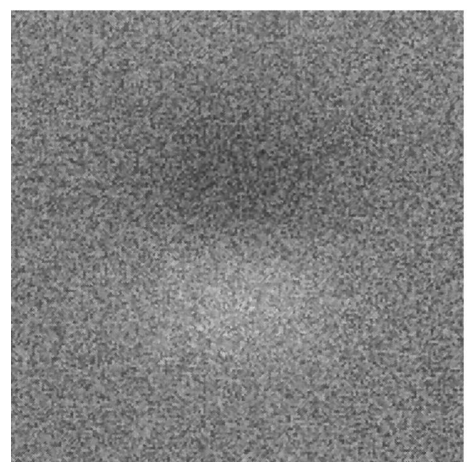

B

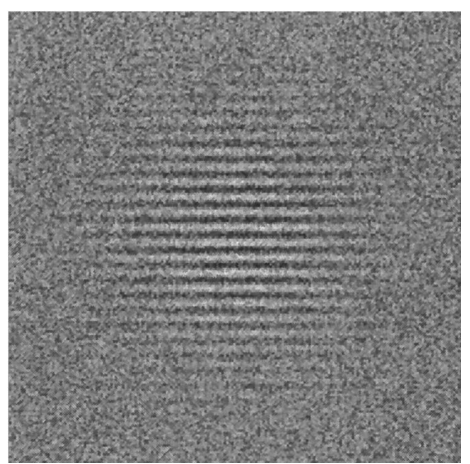

Figure 7. Example displays of 1 (A) and 30 (B) cycles per image, which were used for establishing spatial frequency thresholds. 
contrast that produced $82 \%$ accuracy in response. It is this value that was used to plot the data in Figure 8.

\section{Results and Discussion}

The $\log$ contrast thresholds obtained for S.M. and R.N. are shown in Figure 8, along with the mean of the neurologically intact participants and the boundaries delineating one and two standard deviations on either side of the normal threshold. As is evident from this figure, neither patient showed any difficulty in detecting either low- or high-frequency gratings, performing well within the normal boundaries. When we compare the thresholds of the 2 patients against the neurologically intact participants, there also does not appear to be any clear correlation between their performance on the spatial frequency test and the ability to perceive the local or global form of the stimulus. Both patients performed close to the control mean for the higher frequency displays. S.M., who was able to perceive the global configuration and showed a global advantage in Experiment 2, showed the slightly poorer lowfrequency threshold than the controls and than R.N., whereas this should have been the other way around to account for the hierarchical data. Also, R.N., who processed the hierarchical stimuli locally, had thresholds for the low-spatial frequency that were as good as the best control participant and, therefore, this cannot account for his failure to perceive the global configuration.

Having ruled out the possibility that the discrepancy between the 2 patients in their perception of the hierarchical stimuli was due to differential limitations in analyzing spatial frequency information, we now examine more closely their performance on other tasks of perceptual organization.
Experiment 4: Microgenetic Analysis of the Perceptual Organization of Hierarchical Stimuli

We have assumed, as have others, that the perception of the global configuration of hierarchical stimuli is the outcome of organizational processes involving the grouping of the local elements (e.g., Enns \& Kingstone, 1995; Han et al., 1999a). The patients' inability to apprehend the global form normally, then, serves as evidence that grouping processes may be impaired. In this next experiment, we focused in more specifically on the grouping processes and examined the time course of the perceptual organization of hierarchical stimuli for the 2 patients. This approach, often referred to as a microgenetic approach, involves examining the time course of the development of the percept, rather than just the final outcome of these processes. To conduct this analysis, we adopted the primed matching paradigm, which has been used successfully for this purpose (Kimchi, 1998, 2000). The basic procedure (Beller, 1971) is as follows. Participants view a priming stimulus followed immediately by a pair of test figures, and they must judge, as rapidly and accurately as possible, whether the two test figures are the same as or different from one another. The speed of same responses to the test figures depends on the representational similarity between the prime and the test figures: Responses are faster when the test figures are similar to the prime than when they are dissimilar to it. By varying the duration of the prime, we can tap earlier and later internal representations (Kimchi, 1998, 2000; Sekuler \& Palmer, 1992). Thus, this paradigm enables us to assess implicitly the participant's perceptual representations and the time course of their organization.

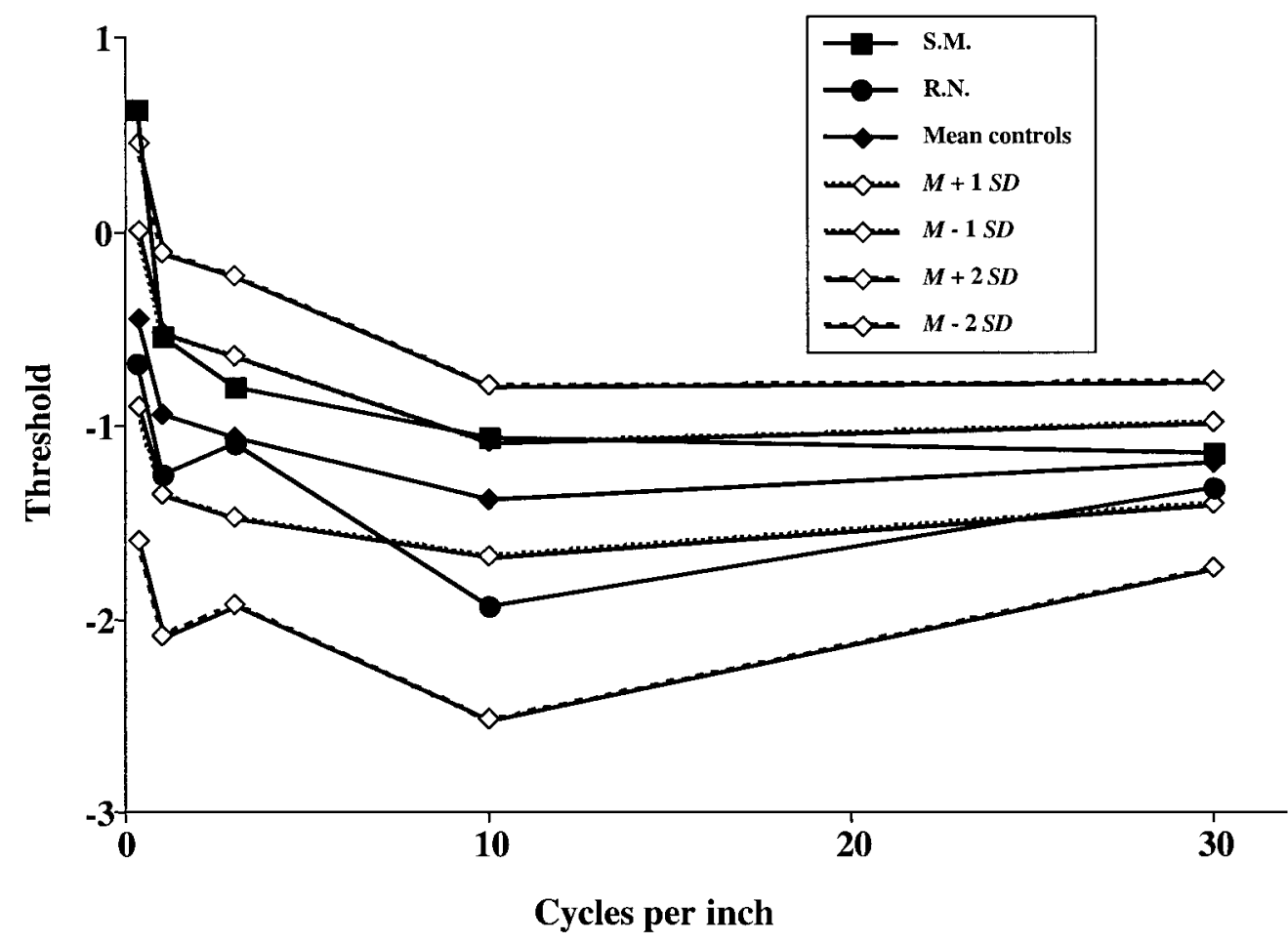

Figure 8. Mean spatial frequency thresholds (reflected as log contrast threshold) and standard deviations as a function of cycles per inch for normal participants and for S.M. and R.N. 
The priming stimuli in the present experiment were few- and many-element patterns, presented at various exposure durations. There were two types of test pairs, defined by their prime-test similarity (see Figure 9): the element-similarity (ES) test pairs in which the test figures were similar to the prime in their local elements but differed in global configuration, and the configuration-similarity (CS) test pairs in which the figures were similar to the prime in global configuration but differed in local elements. Priming effects of the configuration would manifest in shorter correct same RTs for the CS than for the ES test pairs, whereas priming effects of the elements would manifest in shorter same RTs for the ES than for the CS test pairs.

The results for normal participants (Kimchi, 1998) showed that the availability of the elements and the configuration depends on the number and relative size of the elements. Few, relatively large elements are available early on in processing (ES advantage), and they are grouped into a global configuration with time. For manyelement patterns, the configuration is available very early (CS advantage), followed by the individuation of the elements. The question of interest is whether the global configuration of such patterns is available to our patients, and if so, how early in time. In addition, of interest is the effect of number and relative size of elements on the patients' performance in comparison to normal participants.

\section{Method}

Participants. The agnosic patients, S.M. and R.N., participated in this experiment, and we used the data from the normal participants reported in Kimchi (1998) against which to compare their performance.

Apparatus and materials. The experiment, including stimulus generation and presentation, was controlled by a Macintosh G3 laptop. The screen was viewed through a circular aperture $(14 \mathrm{~cm}$ in diameter) of a matte

Prime

Target pair
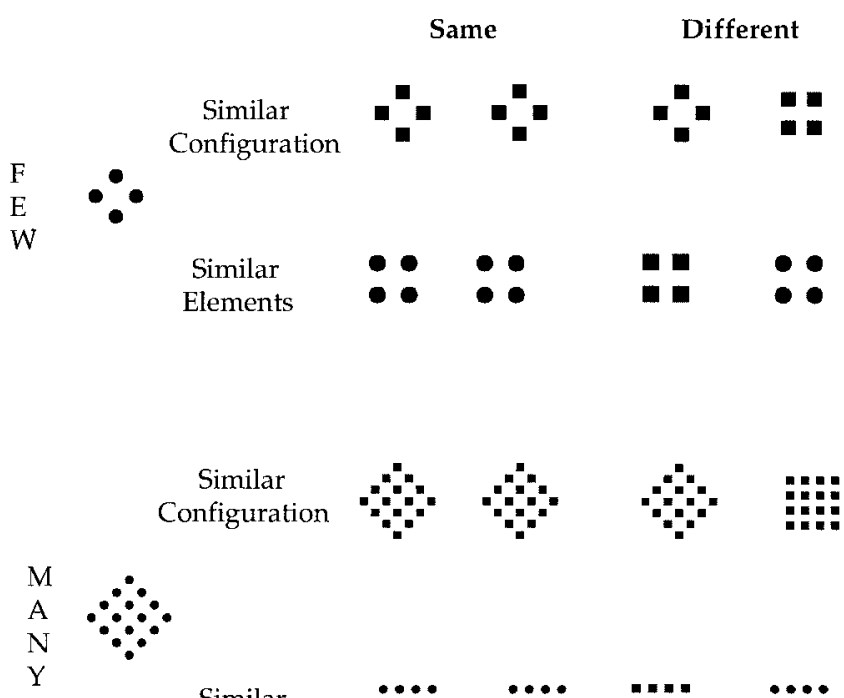

Similar
Configuration<smiles>[IH2]</smiles>

Y

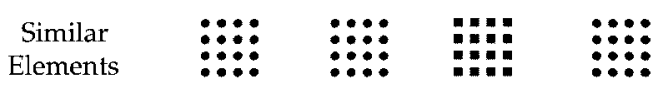

Figure 9. Primed match paradigm. Probes consisting of few and many elements are followed after varying stimulus onset asynchronies by test pairs that require "same" or "different" responses and that are similar to the prime in elements or configuration.

black cardboard sheet so as to exclude any peripheral visual information about the screen or environment. The testing room was dimly lit. Responses were made by pressing one of two response keys, and response times were recorded by the computer. Participants used their dominant hand for the same response key.

The priming stimuli were hierarchical patterns (global diamonds made up of circles) of two types: a few-element pattern and a many-element pattern. The few-element prime was a diamond made of four relatively large circles, and the many-element prime was a diamond made of 16 relatively small circles. Each test stimulus consisted of two hierarchical patterns. There were two types of test pairs, defined by the similarity relations between the test figures and the prime. The test figures in the ES test pairs were similar to the prime in their elements but differed in their configurations. In the CS test pair, the test figures were similar to the prime in their global configurations but differed in their elements. The priming stimuli and the same and different response test pairs are presented in Figure $9 .{ }^{3}$ The global diamond subtended $1.25^{\circ}$ of visual angle, and the global square $0.96^{\circ}$. Each individual circle element subtended $0.36^{\circ}$ (in diameter) in the few-element patterns, and $0.18^{\circ}$ in the many-element patterns. Each individual square element subtended $0.38^{\circ}$ in the fewelement patterns and $0.19^{\circ}$ in the many-element patterns. The distance between the centers of the two stimuli in a test pair was $7 \mathrm{~cm}$.

Design and procedure. The experiment consisted of the factorial combination of four factors in a completely repeated measures design: prime type (few element or many element), prime duration (40, 90, 190, 390, or $690 \mathrm{~ms}$ ), test type (ES, CS), and response (same or different). The fewelement and many-element primes were administered in separate blocks of 160 trials each. All the combinations of the three factors (prime duration, test type, and response) were randomized within block, with each combination occurring on an equal number of trials.

Each trial consisted of the following sequence of events. First, a small fixation dot appeared in the center of the screen for $250 \mathrm{~ms}$, followed by a prime. The presentation time for the priming stimulus was equally and randomly distributed among 40, 90, 190, 390, and $690 \mathrm{~ms}$. Immediately after the presentation of the prime, the test display appeared and stayed on until the participant responded, for a maximum of $3,000 \mathrm{~ms}$. The test display contained two figures presented on either side of the location previously occupied by the prime. At this point, participants had to decide whether the two figures were the same or different and to respond as accurately and quickly as possible using the response keys.

Two sessions were administered, each on a separate day a few weeks apart, with two blocks (one of few-element and one of many-element patterns) in each session. Altogether each patient completed 640 trials. Sixteen practice trials were completed for each of few- and many-element patterns before the experimental trials.

\section{Results and Discussion}

Like the control participants, both S.M. and R.N. performed well on this task, making very few errors (error rate for neurologically intact participants: $3.7 \%$; for S.M.: $4 \%$; and for R.N.: $1 \%$ ). In light of the small number of errors, no further analysis was undertaken and we turned to the RT data. Preliminary analysis of the patients' data indicated no significant effect of session nor an interaction of session with other factors and, hence, we collapsed the data across this variable. Mean correct same RTs for prime-test

\footnotetext{
${ }^{3}$ Kimchi (1998, Experiment 1) used another prime for generalization: a square made up of squares, as well as an $X$, that served as a neutral prime and provided baseline performance for the two test figures. To simplify the experiment for the patients, we used only one prime because no differences between the square and the diamond primes were observed, and we omitted the neutral prime because the performance of the normal participants served as the control for the patients.
} 
similarity (ES, CS) are plotted in Figure 10 as a function of prime duration for each prime type (few-element and many-element patterns) for S.M. and R.N. (B and C, respectively). The results for the neurologically intact participants (from Kimchi, 1998) are used as the benchmark against which to evaluate the patient data and are plotted in Figure 10A.

As can be seen in Figure 10A, few- and many-element patterns produced different patterns of results for neurologically intact participants. For the few-element patterns, responses to ES test pairs were faster than responses to the CS test pairs at 40-, 90-, and 190-ms prime duration, and the difference diminished at the longer prime durations of 390 and $690 \mathrm{~ms}$. For the many-element patterns, responses to CS test pairs were faster than responses to ES at the early durations of 40 and $90 \mathrm{~ms}$. The pattern of RTs reversed at the intermediate durations of 190 and $390 \mathrm{~ms}$ : ES produced faster responses than CS test pairs, and at $690 \mathrm{~ms}$ both element and configuration were available for priming, with a tendency for shorter RTs to CS test pairs (for details, see Kimchi, 1998). These results have been interpreted as suggesting that for normal participants, the elements of the few-element patterns are represented initially, and the global configuration is then consolidated with time. In contrast, in the many-element patterns, there is early representation of the configuration (as in the "forest before the trees"; Navon, 1977), the elements then become individuated, and finally both the configuration and elements are represented and accessible.

The RT data for each patient were analyzed separately by an ANOVA, with trial as a random variable, and prime-test similar- ity, prime type, and prime duration as between-subject variables The results for S.M. showed that responses for few-element patterns were faster by $256 \mathrm{~ms}$ than responses for many-element patterns, $F(1,614)=205.35, p<.0001, M S E=39,885,031$. Responses to ES test pairs were faster than response to CS test pairs, $F(1,614)=30.90, p<.0001, M S E=6,019,258$, but the element advantage was larger for the few-element patterns (which averaged $318 \mathrm{~ms}$ ) than for the many-element patterns (which averaged $78 \mathrm{~ms}$ ), as indicated by the significant interaction between prime type and prime-test similarity, $F(1,614)=11.34$, $p<.0008, M S E=2,202,434$. Furthermore, as can be seen in Figure $10 \mathrm{~B}$, there was a marginally significant interaction between prime-test similarity and duration for the many-element patterns, $F(4,289)=2.00, p<.09, M S E=354,995$, indicating a tendency for a switch to a configuration advantage at the longest prime duration.

The results for R.N. also showed that responses to few-element patterns were faster (by an average of $510 \mathrm{~ms}$ ) than responses to many-element patterns, $F(1,614)=95.36, p<.0001, M S E=$ $10,354,494$. Responses to ES test pairs were faster than to CS test pairs, $F(1,614)=23.75, p<.0001, M S E=2,578,816$, but the element advantage was larger for many-element (which averaged $186 \mathrm{~ms}$ ) than for few-element patterns (which averaged $70 \mathrm{~ms}$ ), as indicated by the significant interaction between prime type and prime-test similarity, $F(1,614)=4.85, p<.03, M S E=526,876$. There was no effect of prime duration on the effect of prime-test similarity.

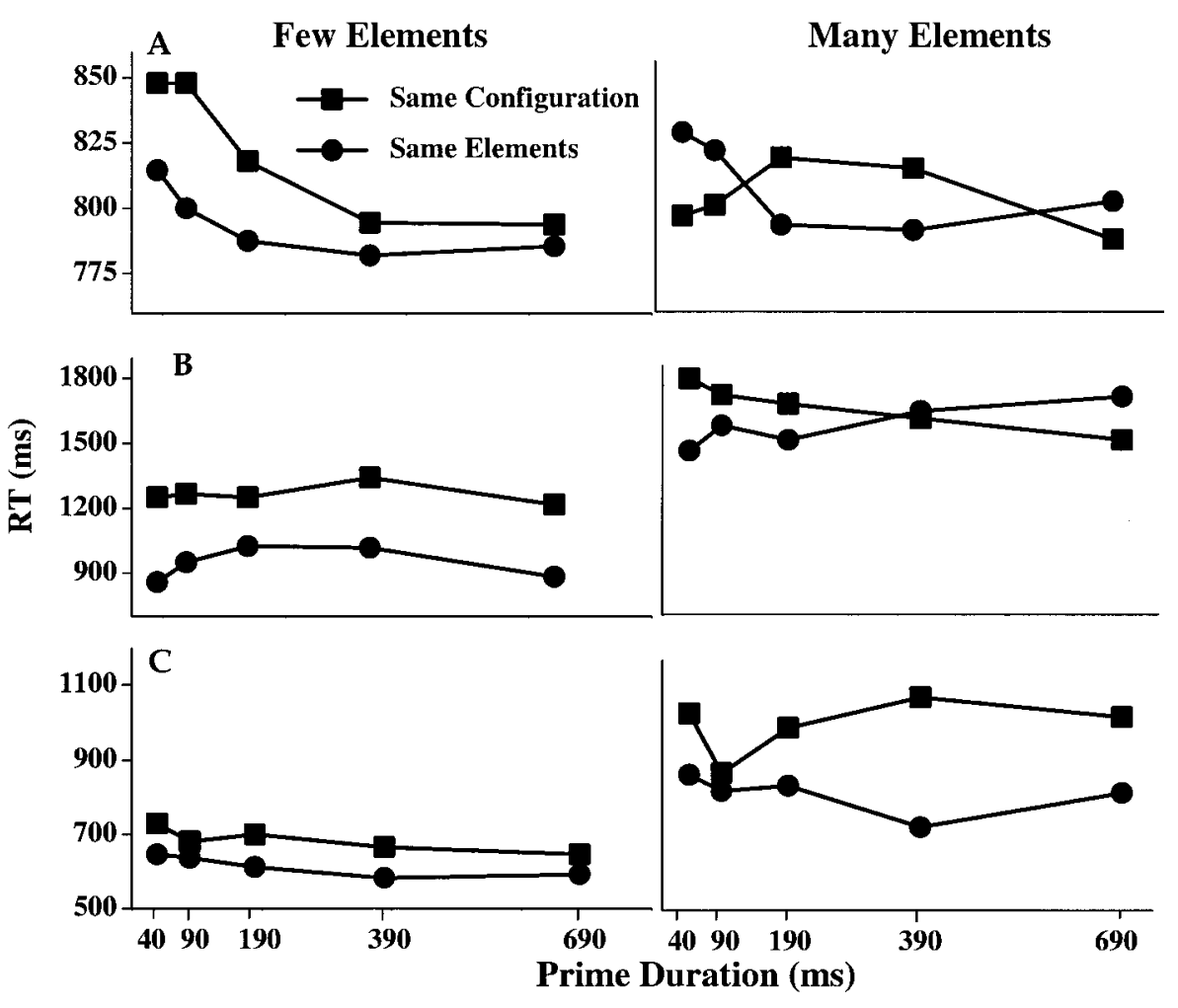

Figure 10. Mean of median correct "same" response times (RTs) for the normal participants (A) and for S.M. (B) and R.N. (C), for few- and many-element displays as a function of prime duration for the two primesimilarity conditions (element similarity and configuration similarity). 
The patients were slower than the normal participants, except in the case of R.N. with the few-element patterns (note the difference of $y$-axis between patients and normal participants in Figure 10). In contrast to the normal participants who responded about equally fast (on average) to few- and many-element patterns, both patients were slower in their responses to many-element than to fewelement patterns, suggesting that it was more difficult for them to handle patterns when the number of elements was increased.

For the few-element patterns, in contrast with the normal participants who seemed to derive the global configuration over time, neither S.M. nor R.N. appeared to be able to derive a global configuration, even at the longest exposure duration of $690 \mathrm{~ms}$. Both patients showed shorter RTs to the ES test pairs, and there was no effect of prime duration on this element advantage. Previous research has suggested that for patterns composed of few, relatively large elements, the local elements are perceived by normal individuals as figural parts of the overall form (Goldmeier, 1936/1972; Kimchi \& Palmer, 1982), and the local elements and the global form are perceptually integral (Kimchi, 1988; Kimchi \& Palmer, 1985). The 2 patients, however, seemed unable to integrate the local elements into a global entity, so that they failed to perceive the local elements as figural parts of an overall form and, rather, perceived them as discrete, unrelated entities.

For the many-element patterns, again in contrast with the normal participants, neither patient exhibited an early advantage for the configuration. Rather, R.N. showed an advantage for the ES test pairs as early as $40 \mathrm{~ms}$, and this element advantage remained fairly unchanged over the entire time course, so that the global configuration was not available to him even at the longest duration of 690 ms. S.M. also did not show any early advantage for the CS test pairs, although he eventually showed a tendency for shorter RTs for CS than ES test pairs at the longest duration of $690 \mathrm{~ms}$, as did normal participants.

It has been suggested (Kimchi, 1998) that for normal participants, many relatively small elements (as in the many-element patterns) are rapidly grouped without the individuation of the elements that occurs later in time. It has also been suggested that such early grouping is functional for a system whose goal is object identification and recognition, because many small elements in close proximity to one another are more likely to be texture elements of a single object rather than discrete objects. No rapid grouping of the many relatively small elements was observed for either patient. Rather, it seems that for both patients, the manyelement patterns were processed initially as discrete elements, hence the longer RTs for many-element relative to few-element patterns.

In addition to the differences between the patients and the normal participants, there were also some differences between R.N. and S.M. First, the difference in RTs for many- and fewelement patterns was larger for R.N. (510 ms) than for S.M. (256 $\mathrm{ms}$ ), reflecting the greater difficulty in processing the manyelement patterns for R.N. than for S.M. ${ }^{4}$ Second, for R.N., the ES advantage for the many-element patterns was larger than for fewelement patterns, whereas the opposite was true for S.M. Third, whereas no effect whatsoever of prime duration on prime-test similarity was observed for R.N., a tendency for a reversal in the relative advantage of ES and CS was observed for S.M. at the longest duration for the many-element patterns.

Taken together, these differences between the patients suggest that in the case of S.M., although there is no evidence for the early rapid grouping of many elements that characterizes normal perception, grouping processes do operate with many elements. Eventually these grouping processes can lead to the perception of the global configuration. This finding is consistent with the results of Experiment 2, in which, with unlimited exposure duration, S.M. showed a global advantage for hierarchical letters, similar to normal participants. Note that previous findings with neurologically intact individuals have shown that grouping is more efficient with an increased number of elements (Bacon \& Egeth, 1991). R.N., on the other hand, seemed unable to group the elements into a global configuration even when conditions and time favor grouping. This clear local advantage in the perception of hierarchical stimuli is consistent with his performance in Experiment 2.

\section{Experiment 5: Integration of Simple Lines Into Contours}

The results of Experiments 2 and 4 suggest that the patients are impaired at grouping multiple elements (presumably by proximity and by similarity) into a global configuration, and R.N. seems to be more impaired at this than S.M. It is interesting that the only instance in which R.N. showed some indication of forming a global configuration was with the $H$ made of $H$ s in Experiment 2, in which collinearity between the local elements could be exploited. In Experiment 5, we examine further the ability of our patients to group line segments into a configuration by collinearity and also by closure. Previous research with normal individuals has demonstrated the perceptual dominance of configuration for disconnected line segments (Kimchi, 1994; Pomerantz \& Pristach, 1989; Rensink \& Enns, 1995), suggesting that even disconnected line segments are grouped into a configuration and that this grouping occurs early and rapidly (Kimchi, 2000; Rensink \& Enns, 1995) and possibly even independently of the number of elements (Donnelly et al., 1991). To map out the microgenesis of line segments into configurations, once again, we adopted the primed matching paradigm and compared the performance of the patients to that of normal individuals (Kimchi, 2000, Experiment 1).

The priming stimuli used in this experiment (see Figure 11) were line configurations (a diamond and a cross) that varied in the connectedness of the line components (no gap, small gap, and large gap) and were presented at various exposure durations. We assumed that the line segments of the cross are likely to be grouped by collinearity, whereas the line segments of the diamond are more likely to be grouped by closure. The relatability theory (Kellman \& Shipley, 1991; Shipley \& Kellman, 1992) that formalizes the Gestalt principle of good continuation suggests that the visual system connects two noncontiguous edges that are relatable, so that the likelihood of seeing a completed figure increases systematically with the size of the angle that must be interpolated, with the $50 \%$ threshold occurring at around $90^{\circ}$. According to this criterion, the cross configuration is characterized by high relatability (an angle of $180^{\circ}$ - collinearity) and the diamond configuration by low relatability (an angle of $90^{\circ}$ ). The diamond configuration, however, possesses closure, whereas the cross does not.

In the experiment, there were two types of same response test pairs defined by the similarity relation between the test figures and

\footnotetext{
${ }^{4}$ We have to be somewhat cautious about this statement in light of the fact that R.N.'s RTs to the few-element patterns were rather short (almost shorter than those of the normal participants).
} 


\section{Prime}

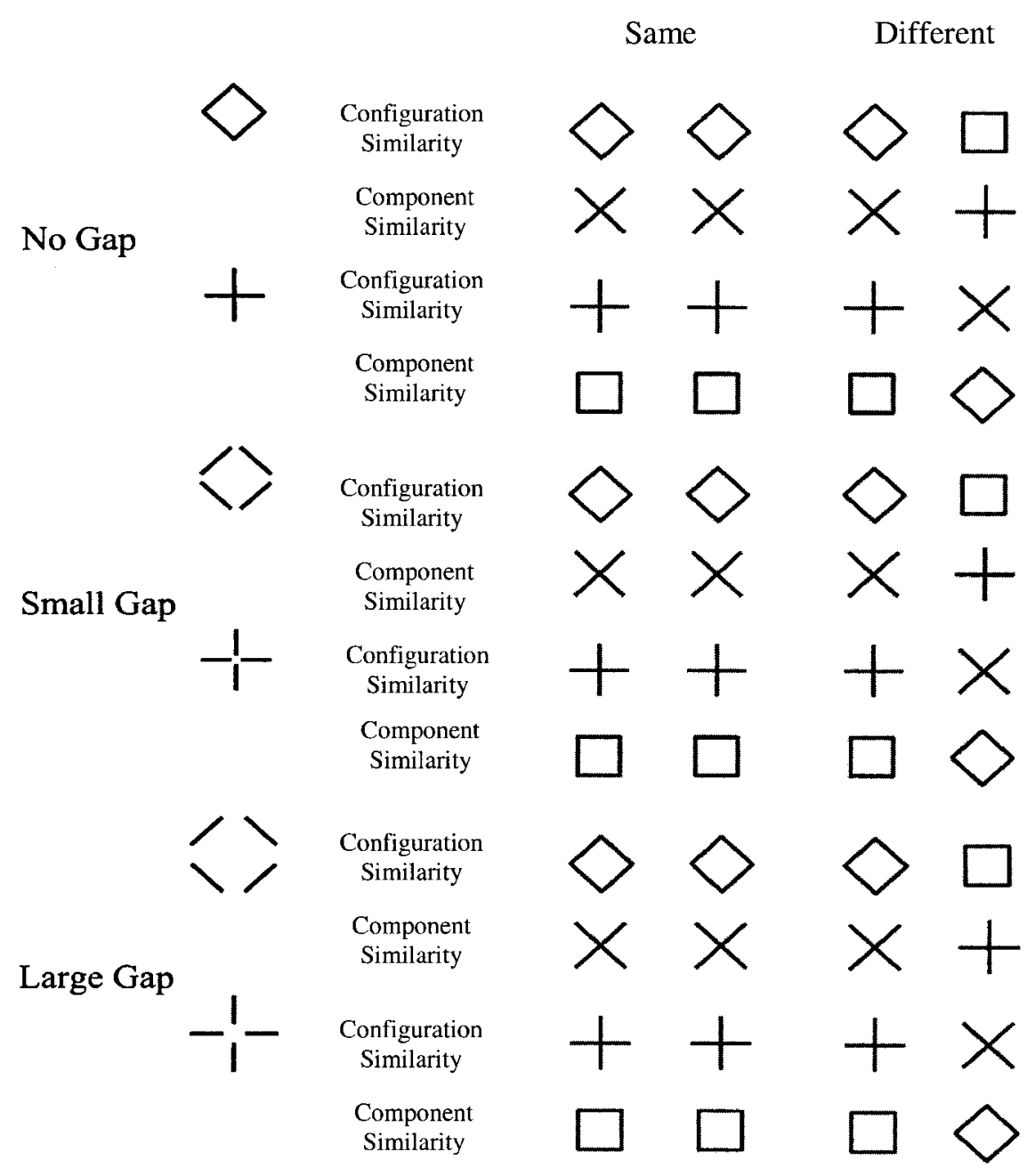

Figure 11. Priming stimuli and the "same" and "different" response pairs used in the no-gap condition, the small-gap condition, and the large-gap condition.

the prime. The figures in the configuration similarity test pair were similar to the prime in both configuration and line components, whereas the figures in the component similarity test pair were similar to the prime in lines but dissimilar in configuration. For example, when the prime was a diamond (irrespective of gap size), and the test pair was a pair of diamonds, prime-test similarity reflected configuration similarity, whereas when the test pair was two $X \mathrm{~s}$, prime-test similarity was component similarity. ${ }^{5}$ For this set of stimuli, priming effects of the configuration would manifest in shorter correct same RTs for the configuration similarity than for the component similarity test pairs. No difference in RT due to component priming between the two types of test pairs was expected because both types of test pairs were similar to the prime in line components. The results for the neurologically intact participants showed early availability of the configuration that was particularly strong for the no-gap and the small-gap conditions. The question of interest is whether the global configuration of such patterns was available to our patients, and if so, how early in time in comparison to neurologically intact participants.

\section{Method}

Participants. The 2 patients, S.M. and R.N., participated in this experiment. We used the data from the neurologically intact participants reported in Kimchi (2000, Experiment 1) against which to compare their performance.

Apparatus and materials. The apparatus was the same as that used in Experiment 4. The priming stimuli were line configurations (a diamond and a cross) that varied in connectedness between their line components (no gap, small gap, and large gap). The figures in the configuration similarity test pair were similar to the prime in both configuration and line components, and the figures in the component similarity test pair were

\footnotetext{
${ }^{5}$ In addition to the diamond and cross prime, Kimchi (2000, Experiment 1) used a random array of dots for which prime-test similarity was considered neutral and which served as a baseline condition. To simplify the experiment for the patients, we omitted the neutral prime because the performance of the normal participants served as the control for the patients.
} 
similar to the prime in lines but dissimilar in configuration. All test figures were connected configurations (see Figure 11).

Each individual line subtended $1.43^{\circ}$ in length for the diamond and the cross primes for all primes and test figures. The connected diamond subtended about $2.02^{\circ} \times 2.02^{\circ}$, and the connected cross subtended $2.86^{\circ} \times$ $2.86^{\circ}$. The gaps between the lines subtended $0.29^{\circ}$ each in the small-gap condition and $1^{\circ}$ each in the large-gap condition. The size of the test figures was identical in all gap conditions. The distance between the centers of the two stimuli in a test pair was $7 \mathrm{~cm}$. The figures in the different response test pairs appeared equally often in each of the two possible locations.

Design and procedure. The experiment consisted of the factorial combination of five factors in a completely repeated measures design: gap (no gap, small gap, large gap), prime type (diamond or cross), prime duration $(40,90,190$, or $390 \mathrm{~ms})$, prime-test similarity (component similarity or configuration similarity), and response (same or different). The three different gap conditions constituted a between-blocks manipulation for the neurologically intact participants. All the combinations of the four other factors (prime type, prime duration, test type, and response) were randomized within block, with each combination occurring on an equal number of trials. For each gap condition, there were six blocks of 160 experimental trials each, preceded by a block of 15 practice trials. The patients completed two sessions, a few weeks apart, with three blocks in each session. The procedure was the same as in Experiment 4.

\section{Results and Discussion}

Like the neurologically intact participants, S.M. and R.N. made very few errors on this task (error rate for normal participants: $1.4 \%$; for S.M.: $0.2 \%$; and for R.N.: $0.7 \%$ ). In light of the small number of errors, no further analysis was undertaken, and we turned to the RT data. Preliminary analysis of the patients' data indicated no significant effect of session nor an interaction of session with other factors, and hence, we collapsed the data across this variable. Mean correct same RTs for each prime-test similarity relation (component similarity, configuration similarity) are plotted in Figure 12 as a function of prime duration for each gap condition for the two prime types (diamond and cross) for S.M. and R.N. (Figures 12B and 12C, respectively). The results for the neurologically intact participants are used as the benchmark against which to evaluate the patient data and are plotted in Figure 12A. Analyses of the correct same RTs for the neurologically intact participants (Kimchi, 2000, Experiment 1) showed that prime type (diamond or cross) did not interact significantly with priming effects, prime duration, and gap condition, and therefore, the data for the neurologically intact participants were collapsed across prime type.

As can be seen in Figure 12A, for neurologically intact participants, configuration similarity produced shorter RTs than component similarity as early as $40 \mathrm{~ms}$ for the no-gap and the small-gap conditions, and there was no effect of prime duration on this configuration advantage. No significant difference between configuration similarity and component similarity was observed for the large-gap condition (for details, see Kimchi, 2000, Experiment 1). ${ }^{6}$ These results have been interpreted as suggesting that for neurologically intact individuals, disconnected line segments are rapidly organized into configurations, provided collinearity (the cross prime) or closure (the diamond prime) is present. Strong proximity between the line segments (as in the no-gap and smallgap conditions) facilitates grouping by closure or collinearity more than does weak proximity (as in the large-gap condition), but because a configuration advantage was observed early both for the connected and the small-gap disconnected primes, connectedness does not seem to be necessary for rapid grouping.

The RT data for each patient were analyzed separately by an ANOVA, with trial as the random variable, and prime-test similarity, prime type, prime duration, and gap condition as betweensubject variables.

The results for S.M. (Figure 12B) showed a significant effect of prime type with shorter RTs for crosses than diamonds, $F(1$, $1360)=18.83, p<.0001, M S E=1,135,106$, and a significant effect of duration, $F(1,1360)=7.81, p<.0001, M S E=468,901$, with shorter RTs as duration increased. There was also a significant effect of prime-test similarity, $F(1,1360)=17.27, p<$ $.0001, M S E=1,036,014$, that interacted with prime type, $F(1$, $1360)=22.95, p<.0001, M S E=1,386,165$. As can be seen in Figure 12B, RTs for configuration similarity were significantly shorter (by an average of $117 \mathrm{~ms}$ ) than RTs for component similarity for the diamond prime, but no difference between the two test types was observed for the cross prime. The configuration advantage decreased with an increase in gap size, as indicated by the significant interaction between prime-test similarity and gap condition, $F(2,1360)=3.28, p<.05, M S E=192,494$, and it increased with prime duration, as indicated by the significant interaction between prime-test similarity and prime duration, $F(3$, $1360)=3.99, p<.01, M S E=240,689$. No other interactions were significant.

The results for R.N. showed a significant effect of prime-test similarity, $F(1,1381)=13.22, p<.001, M S E=294,950$, that varied with gap condition, $F(2,1381)=3.13, p<.05, M S E=$ 69,544 . There was a significant advantage for configuration similarity over line similarity for the no-gap condition (which averaged $51 \mathrm{~ms}$ ) and the small-gap condition (which averaged $33 \mathrm{~ms}$ ), roughly equal across the two prime types, but no significant difference between configuration similarity and component similarity was observed for the large-gap condition. Like S.M., R.N.'s RTs were shorter when the prime was a cross than a diamond, $F(1$, 1381) $=34.94, p<.0001, M S E=781,087$, but prime type did not interact significantly with prime-test similarity, prime duration, and gap condition.

R.N. showed a priming effect of the configuration both for the diamond and for the cross primes that decreased with gap size. As long as the gaps between the line components were relatively small (i.e., relatively strong proximity), he was able to integrate them either by collinearity or by closure. S.M., on the other hand, showed a priming effect of the configuration for the diamond prime but no priming effect for the cross prime. Because S.M.'s responses, like R.N.'s, were faster for the cross than for the diamond prime, it is unlikely that the absence of a configuration advantage for the cross indicates that S.M. could not use collinearity for grouping. Rather, this finding may have resulted from S.M.'s high sensitivity to closure. Given that the component similarity test pair for the cross includes two squares and the configuration similarity test pair includes two crosses (see Figure 11), it is possible that although responses to the configuration similarity

\footnotetext{
${ }^{6}$ It is important to note that when RTs for the component similarity test pairs were compared with baseline performance, no facilitation for the component similarity test pair was observed even for the large-gap condition, suggesting that even under relatively weak proximity between the lines, there was no relative dominance of the component lines.
} 

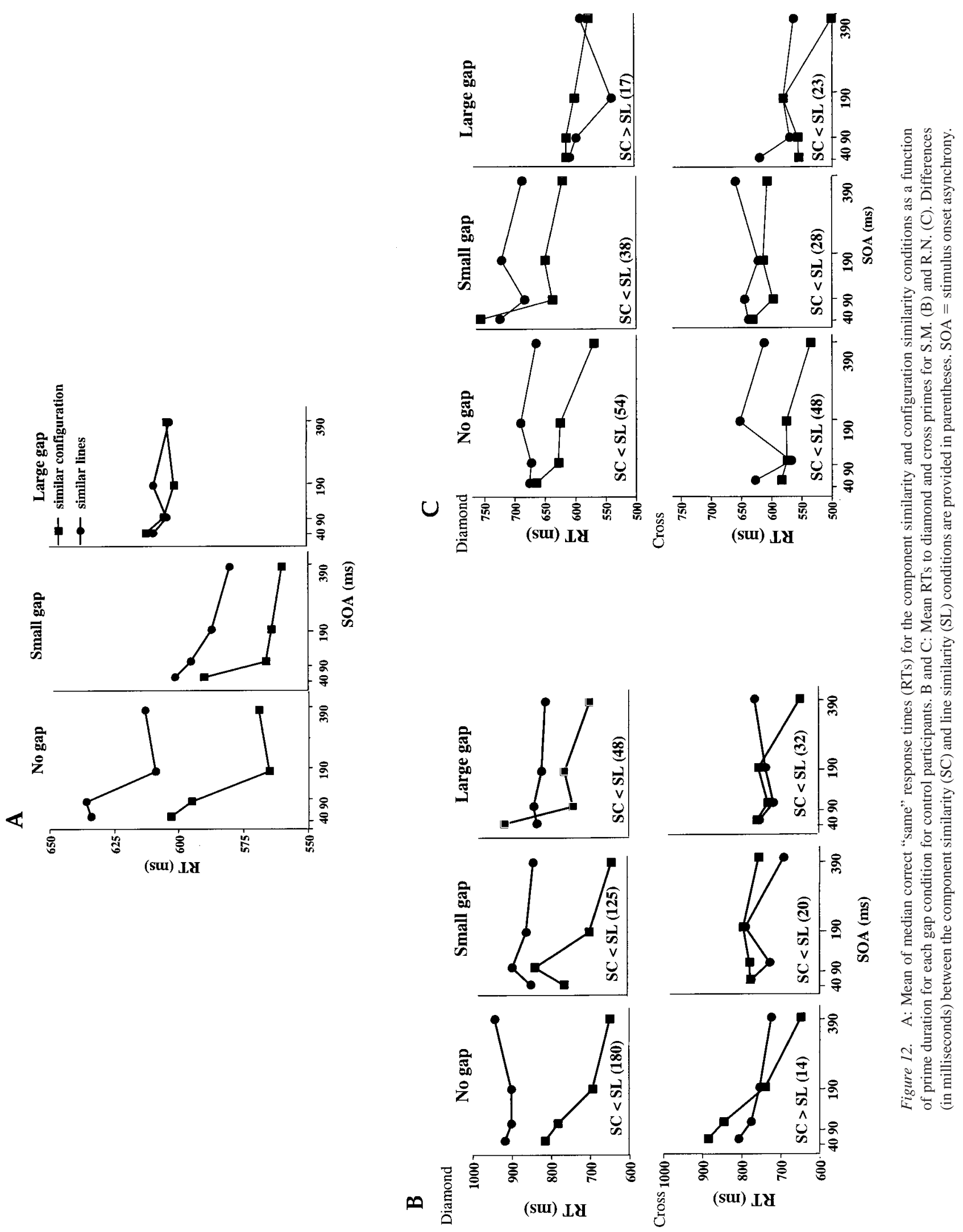
test pairs were facilitated because of prime-test similarity, responses to the component similarity test pairs were facilitated because of the closure present in the square test figures, and as a result, no difference between the two types of test pairs was obtained. It is not the case, then, that S.M. was impaired at grouping by collinearity whereas R.N. was not, but rather that S.M. was more sensitive than was R.N. to closure. Further support for the claim that S.M. was more sensitive to closure than was R.N. comes from the finding that the configuration advantage for the diamond was larger for S.M. (180, 125, and $48 \mathrm{~ms}$, for the no-gap, small-gap, and large-gap conditions, respectively) than for R.N. (54, 38, and $17 \mathrm{~ms}$, for the no-gap, small-gap, and large-gap conditions, respectively; see Figure 12), and furthermore, R.N., unlike S.M., did not show any configuration advantage but rather an element advantage for the large-gap condition. That is, strong proximity facilitated grouping by closure for R.N., whereas for S.M., closure was strong enough to override weak proximity. It is interesting that the performance of the neurologically intact participants in the neutral prime condition also showed faster responses to the pairs of squares than to the pairs of crosses (Kimchi, 2000, Experiment 1), suggesting a sensitivity of the neurologically intact participants to the property of closure.

To rule out the possibility that the difference between R.N. and S.M. in their responses to the cross prime is due to a difference in their ability to exploit collinearity, we now compare their performance in an elementary contour interpolation task.

\section{Experiment 6: Contour Interpolation}

To test contour detection thresholds, we used a set of cards containing displays of a smoothly aligned, closed path of Gabor elements embedded in a random array of Gabor elements of the same spatial frequency and contrast, devised by Pennefather, Chandna, Kovács, Polat, and Norcia (1999). In this test, cards containing the displays are presented individually to the participant, who is required to indicate the location of the contour formed by the Gabor patches. The critical manipulation or parameter, called delta, is the spacing between the adjacent elements in the background relative to the spacing between neighboring elements along the contour. The delta ranges from 1.2 (card 2_1) to 0.5 (card 2_15) in steps of 0.05 (examples of these displays are presented in Figure 13). This parameter expresses relative noise density, and reflects, in a way, signal-to-noise ratio so that the smaller the delta value, the easier detection is. It has also been suggested that as delta decreases, long-range spatial interactions of oriented features, presumably mediated by low-level areas of visual cortex, are more involved. Given that early visual areas are preserved in both patients, we expected them both to perform normally. If they did so and there was no difference between them, this would further indicate that they both could exploit collinearity as a grouping heuristic. Establishing contour detection thresholds using this method has been successfully achieved previously with various pathological populations (Kovács, Polat, Pennefather, Chandna, \& Norcia, 2000).

\section{Method}

Participants. S.M. and R.N. participated in this study, and we used the data from the neurologically intact participants reported in Kovács et al. (2000) against which to compare their thresholds.

Stimuli and procedure. Cards of increasing delta were presented individually to a participant for an unlimited exposure duration, and the participant was required to indicate the location of the contour. A staircase procedure was used to establish threshold. For more details, the reader is referred to Kovács et al. (2000).

\section{Results and Discussion}

Both S.M. and R.N. completed this task easily and effortlessly. It is important to note that both attained thresholds within normal limits, with deltas of 0.60 and 0.65 , respectively. The normal is around 0.70 (Kovács et al., 2000). It is interesting to note, at this point, that patient H.J.A. also performed well on the present task,
A

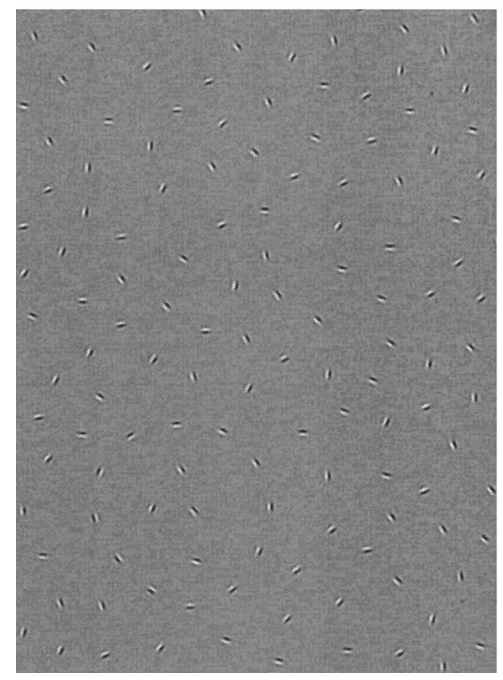

B

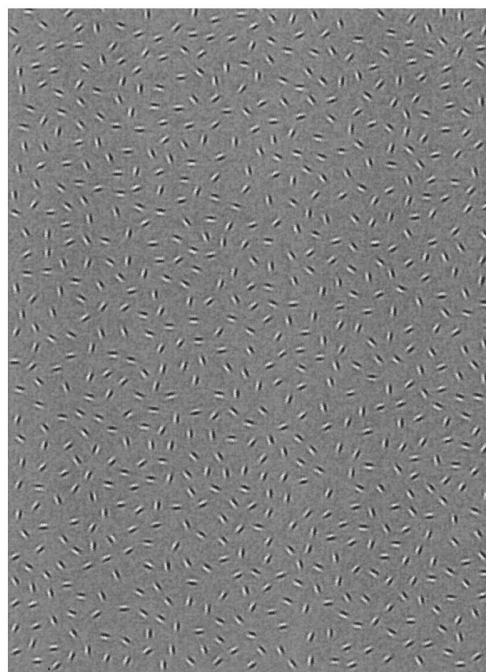

Figure 13. Examples of displays used in the experiments presented in Kovács et al. (2000) of contours made of local Gabor units. A: Easy. B: Difficult. Printed with permission of Ilona Kovács. 
obtaining a threshold of 0.65 (Giersch, Humphreys, Boucart, \& Kovács, 2000). These findings indicate that both our patients have a normal ability to integrate collinear elements into contours and that there is no obvious difference between them in this ability. These data can explain the finding of faster responses for the cross prime (presumably grouping by collinearity) than for the diamond prime (presumably grouping by closure) that were observed in the previous experiment for both patients. Further support for their ability to integrate collinear elements comes from the results of Experiment 2, in which even R.N., who was generally unable to derive the global configuration of the many-element patterns, was able to do so in the case of an $H$ made of $H$ s. Furthermore, the present findings support the claim that the difference between R.N. and S.M. for the cross prime in Experiment 5 is unlikely to have arisen from a differential sensitivity to collinearity, but rather from a difference in their sensitivity to closure: S.M. is more sensitive to closure than R.N. is.

The consistency in performance across the patients (both ours and H.J.A.) in the contour interpolation task endorses the notion that the integration of contours in a task such as this likely relies on visual processes mediated by earlier or lower level regions of visual cortex and that these areas are preserved in integrative agnosic patients.

\section{Experiment 7: Grouping by Proximity and by Similarity in Luminance}

We assumed that in the hierarchical stimuli used in Experiments 2,4 , and 5, the local elements were grouped into a global configuration by proximity and/or by similarity, and the inability of the patients to apprehend the global configuration reflected an impairment in grouping. However, as we mentioned in the introduction, perceptual organization is thought to involve two operations: element clustering, which determines which elements belong together, and shape formation or configuring, which determines the shape of the grouped elements (Rock, 1986; Trick \& Enns, 1997). It is possible then, that our patients were not impaired in clustering but rather in shape formation or configuring. That is, it is possible that they were able to group the elements of the hierarchical stimuli into a unit but were impaired in shape formation and configuring so that the unit was not organized for them into a whole that has unique qualities such as shape. To explore this possibility, we examined the performance of the 2 patients in simple grouping tasks: grouping into columns or rows by proximity and by similarity in luminance.

\section{Method}

Participants. S.M. and R.N. participated along with 4 neurologically intact control participants (mean age 19.2 years), who completed this study as part of course credit at Carnegie Mellon University.

Stimuli and procedure. A display consisting of small circles, each 4 $\mathrm{mm}$ in diameter, appeared centered on a computer screen (see Figure 14 for examples). In the proximity condition, the display contained 32 solid black circles, and the distance between them horizontally or vertically was manipulated to yield an organization of either rows or columns, respectively. The distance was either 4 or $8 \mathrm{~mm}$ from the center of one circle to the next, and, depending on the distance, the arrangement obeyed a row or column organization. In the similarity condition, the elements were solid black and white circles, equally distant $(4 \mathrm{~mm})$, and the organization was determined by the alternation of the two colors, either in rows or columns.
A

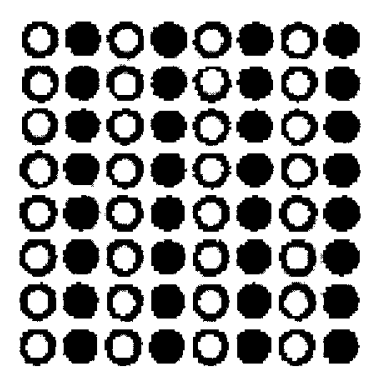

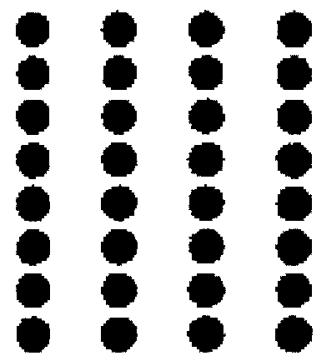

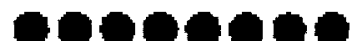
$\bullet \bullet \bullet \bullet \bullet \bullet \bullet \bullet$ $\bullet \bullet \bullet \bullet \bullet \bullet \bullet \bullet$ $\bullet \bullet \bullet \bullet \bullet \bullet \bullet \bullet$

Figure 14. Arrangement of dots into columns (A) and rows (B) for grouping by similarity and by proximity.

The participants were instructed to indicate, for each display, whether an arrangement of rows or columns was present. There were 50 trials in each of the two organization conditions, rows or columns, and we measured both accuracy and RT.

\section{Results and Discussion}

Both patients performed well on this task as did the neurologically intact controls (correct response rate for controls: $93.3 \%$ in both conditions). S.M. was correct $90 \%$ and $94 \%$ of the time in the proximity and similarity conditions, respectively, and R.N. was correct $100 \%$ of the time in both conditions. That is, when proximity favored an organization of rows, the patients perceived rows, and when it favored an organization of columns, they perceived columns. The same was true when organization was based on similarity in luminance. These findings indicate that both patients were sensitive to grouping by proximity and by similarity in luminance and were able to determine the orientation of the grouped elements. If anything, whereas R.N. scored perfectly, S.M. made a few errors, possibly because of a speed-accuracy tradeoff as S.M. was much faster (proximity RT: $603 \mathrm{~ms}$; similarity RT: $659 \mathrm{~ms}$ ) than R.N. was (proximity RT: $917 \mathrm{~ms}$; similarity RT: $862 \mathrm{~ms})$.

However, grouping by proximity and by similarity may not suffice for deriving the shape of the grouped elements. It is interesting that Razpurker-Apfeld and Kimchi (2003) found that grouping by similarity of color into columns or rows occurred more quickly than grouping into arrows or triangles and that the former but not the latter occurred under conditions of inattention. This finding suggests that grouping is not a single process even when it is based on the same heuristic, but, rather, it involves operations that differ in their time course and attentional demands.

The findings of the present experiment suggest that the difficulty of our patients, and in particular R.N., to apprehend the 
global configuration of hierarchical stimuli was not due to an impairment in simple grouping (i.e., in the sense of what goes with what) but presumably to an impairment in the ability to apprehend the interrelationships of the elements and to derive the emergent structure or shape.

\section{General Discussion}

The goal of this article has been to explore the psychological and neural processes involved in deriving structure and coherence from visual input. Many traditional theories of visual perception have assumed that perceptual organization processes operate early and preattentively to deliver candidate units for further processing, such as object identification. These theories make no attempt to distinguish in detail between the different perceptual organization processes nor do they evaluate the relative contribution of the different processes to object recognition. To address these issues, we have investigated the behavior of 2 individuals with acquired integrative visual object agnosia on tasks of object recognition and perceptual organization, with a specific emphasis on grouping elements into global forms. By understanding how the system breaks down and how a perceptual organization deficit is related to impaired object recognition, we hoped to obtain insight into the normal processes of perceptual organization and object identification.

When required to integrate many small elements into a global configuration, S.M. but not R.N. was able to derive the global form although he required more time to do so than did the normal participants. A microgenetic analysis of this integration process confirmed that given enough time and sufficient data-driven support, S.M. was eventually able to derive the global form. It is important to note that neurologically intact perceivers unify a multielement stimulus early and quickly, reflecting their spontaneous bias to deal with such a stimulus as a unit rather than as disparate components, and only later do they individuate the elements. S.M. did not show this early and fast grouping of the many elements, and only with time was he able to laboriously derive a global form. Even under optimal circumstances, R.N. failed to derive the global form from the image. When the stimulus was composed of only a few relatively large elements, neither patient was able to extract a global structure. Under these conditions, neurologically intact participants could apprehend the global structure despite the relative salience of the individual elements, and the global configuration became further consolidated with time. Note that the differences between the 2 patients and the difference between them and the neurologically intact participants in apprehending the global configuration of hierarchical stimuli cannot be attributed to a differential sensitivity to low-spatial-frequency information, as both patients displayed spatial frequency threshold functions within normal limits.

In an investigation of the time course of the ability to group simple line segments into configurations by collinearity and by closure, we found that both patients were able to exploit these properties early on, as is also true of neurologically intact participants. However, S.M. was more sensitive to closure than was R.N. Strong proximity facilitated grouping by closure for R.N., but S.M. was able to group by closure even when proximity was weak. We should note that R.N.'s performance here might even be mediated by collinearity at its limits (relatability at $90^{\circ}$ ), indicating that he might be even less sensitive to closure than we have suggested. In a task that was designed specifically to evaluate the ability to integrate collinear elements into simple contours, both S.M. and R.N. performed like normal perceivers and did not differ from one another.

The final result was that when presented with classic, simple gestalt displays requiring grouping into rows or columns, both patients were able to group by proximity and by similarity of luminance and did not differ from one another nor from the neurologically intact participants.

In sum, both patients were able to group collinear elements into a contour, to group elements into simple rows or columns by proximity and by similarity in luminance or color, and to group simple line segments into simple configurations by closure. It is important to note, however, that although the basic grouping abilities of both patients seemed intact under simple conditions, they nevertheless encountered difficulties under more difficult conditions as in segmenting overlapping shapes. In contrast with the seemingly intact basic grouping, there was a significant impairment in both patients in deriving global structure and apprehending a multielement stimulus as a whole with a specific shape, and the impairment was more marked in R.N. than in S.M. R.N. failed to derive a global structure even under the most favorable conditions and unlimited time and was also less sensitive to closure than was S.M. A critical point here is that the patients differed from each other in the severity of their object recognition deficit, with R.N. performing significantly more poorly than S.M. in both accuracy and latency.

We now discuss these findings in light of our original questions, namely, the differences between various processes of perceptual organization, the relative contribution of these different processes to object recognition, and the neural systems subserving these mechanisms.

The first important conclusion is that not all organizational processes are created equal. Clearly, grouping by collinearity, proximity, and similarity by luminance or color was easily and equally well achieved by the patients, whereas this was not the case for grouping by closure. The relative preservation of grouping by collinearity is also evident in two other agnosic patients: H.J.A. (Giersch et al., 2000) and N.M. (Ricci et al., 1999), who were impaired at integrating low-level elements into a whole, but, nevertheless, were able to extract contours from an image (see also patient F.G.P., Kartsounis \& Warrington, 1991). The present data also clearly show that although the patients were sensitive to basic grouping, they were not equally able to derive a global structure and shape, suggesting that they might be impaired (and to different degrees) in configuring or shape formation.

The differential sensitivity to different forms of grouping is consistent with the idea that some perceptual organization processes may precede others; for example, some processes operate on fairly local components, such as edges, and map onto basic neurophysiological interactions quite early in the visual pathway (Kellman, 2000; Kovács, Kozma, Feher, \& Benedek, 1999; Shipley \& Kellman, 1992). This also fits well with recent neuroimaging and neurophysiological work (Lamme \& Roelfsema, 2000; Lee, in press; Sugita, 1999; Westheimer, 1999) suggesting that the ability to interpolate across discrete collinear elements arises from the lateral connections and long-range interactions in early (V1 and V2) visual cortex. Time constants associated with the V1 and V2 operations have been estimated at 45 to $50 \mathrm{~ms}$ and 70 to $90 \mathrm{~ms}$ in V1 and V2, respectively (Doniger et al., 2000; von der Heydt \& 
Peterhans, 1989). Unfortunately, as is often the case in neuropsychological investigations and as is true in our case too, the lesion localization in our patients is not precise enough to yield definitive evidence for the neural structures that are involved, but our results are consistent with the neurophysiological and imaging findings. We know, for instance, that the early visual areas were preserved in S.M. and although not definitely established, were likely intact in R.N., too, given the absence of low-level deficits. It is these preserved visual regions that probably mediated the patients' ability to exploit collinearity and grouping by proximity and similarity in luminance. An obvious future question to be addressed concerns the neural substrate for these organization processes. Ideally, contrasting a group of integrative agnosia patients with lesions in a particular area with a group of control patients with lesions in a different area would present stronger evidence for the brainbehavior correspondences in which we are interested.

In contrast with these rather simple forms of grouping, other forms of organization had more global influences, as in the case of closure and deriving a structured whole, and these more complex forms were probably mediated by more anterior regions of the visual system. Some evidence to support this claim, for example, comes from a recent high-density, event-related potential study (Doniger et al., 2000) in which the amount of visual information in the image was incrementally increased with each subsequent presentation. The critical result was the existence of a bilateral occipitotemporal negative potential that tracked the amount of closure in the image; activation did not manifest in an all-or-none fashion at the point of closure but rather built incrementally over a number of preidentification levels. This finding suggests that this region is involved in the computation of closure rather than just registering its presence. It is important to note that the peak of activation in this region occurred at approximately $290 \mathrm{~ms}$, much later than the estimated onset of V1 or V2 activity. That perceptual closure is subserved by ventral occipitotemporal areas is also supported by recent hemodynamic and metabolic data (Gerlach et al., 2002) showing that the inferior occipital gyri (perhaps even including area V2) are involved in the integration of visual elements into perceptual wholes, irrespective of whether the wholes were familiar objects or not (see Georgopoulos et al., 2001, for similar evidence; and Gauthier \& Tarr, 2002, for behavioral evidence on different forms of configuring and associated neural substrates).

The hypothesis that we have put forward entails that some organization processes precede others, and we have linked these to brain structures on a continuum from more posterior to anterior regions. We do not, however, propose that the system operates in a purely serial and feedforward fashion. There is now ample evidence for bidirectional connectivity and mutual bottom-up and top-down reciprocity (Bullier \& Nowak, 1995; Lee, in press; Zhou, Friedman, \& von der Heydt, 2000) and the fact that processing operates in a cascaded and interactive fashion in the visual system. We do suggest, however, that there is a temporal advantage for some processes over others, and the order in which these processes take place follows a posterior-anterior brain organization.

The finding that S.M. and R.N. are similar to one another in more basic, presumably low-level grouping operations but show marked differences in their ability to derive a global form from a multielement display strongly suggests that perceptual organization involves not only grouping in the sense of element clustering, but also presumably higher level configuring and shape formation.
It is in these more configural forms of grouping that the 2 patients differed from one another and in which R.N. was more impaired than was S.M. This distinction we have made between grouping and shape formation or configuring may also help to clarify some confusion found in the literature on perceptual organization. For example, understanding the attentional demands of perceptual organization may depend on whether we refer to grouping (i.e., element clustering) or to configuring (shape formation). The former is more likely to occur under conditions of inattention than the latter, and a failure to distinguish between the two organizational processes may lead to seemingly conflicting results. Also, when a task that is designed to assess grouping performance also requires shape formation, caution is necessary in interpretation. For example, Ricci et al. (1999) reported that patient N.M. was impaired in grouping (by luminance, by color, and by line orientation). However, the test that was administered to examine N.M.'s grouping ability required her to identify a hierarchical letter embedded in a background of elements. Clearly, grouping alone (i.e., determining which elements belong together) is not sufficient for deriving the structure or the shape of the grouped elements in this case, and shape formation is also necessary. It is unclear, then, whether N.M. was impaired in grouping or in shape formation or in both.

The differences between the patients in their sensitivity to closure and their ability in configuring and shape formation parallels the difference between their object recognition performance in that R.N. was more impaired than S.M. was in both accuracy and speed of object identification. Clearly both patients were able to group collinear elements into a contour, but there is more to an object than a contour, and it appears that it is the higher level processes that are critical for object recognition. This is supported by R.N.'s object recognition errors, which reflect his inability to derive form with extent and surfaces, and his reliance on simple contours to extract the outline of the shape. For example, when shown a black and white line drawing of a tie, he identified it as "string" and on another occasion referred to a drawing of a nose as "string." In contrast to simple contours, objects are considered to be complex wholes: They have contours, but they also have extent, closure, and internal structure (Feldman, 1999, 2000; Sanocki \& Sellers, 2001). Indeed, some studies have shown that under some circumstances, the global shape (and perhaps some surface properties, as revealed, for example, when silhouettes are used as stimuli) may automatically induce activation of object representations (Boucart \& Humphreys, 1992b; Boucart, Humphreys, \& Lorenceau, 1995; Dell'Acqua, Job, \& Grainger, 2001).

At the same time, it is conceivable that there are circumstances in which certain lower level grouping may suffice for object recognition; for example, when grouping by collinearity provides contours, and the object is easily recognizable from the contours. It may also be the case that object recognition may occur without a full apprehension of the whole (Davidoff \& Warrington, 1999). For example, a real, familiar object may be recognized by a distinctive feature or part that is uniquely diagnostic of the object's identity. Thus, we do not claim that all forms of grouping and configuring (or shape formation) are always necessary for object recognition, but rather that simple grouping is not sufficient for object recognition, whereas shape formation and configuring are critical for it.

Before concluding, we need to consider a final issue that emerges from the present findings and that concerns the relation- 
ship between spatial frequency analysis, performance on tasks evaluating global or local processing, and the relationship between these and the two cerebral hemispheres. With regard to spatial frequency and global or local processing, one view has assumed a direct relationship between spatial frequency filters and global or local bias: High-spatial-frequency information supports the local analysis of the image, and low-spatial-frequency information supports the global analysis of the image (Hughes et al., 1990; Shulman et al., 1986; Shulman \& Wilson, 1987). The data from our 2 patients challenge this assumption. Both patients exhibited normal spatial frequency thresholds in both the high- and lowfrequency range, yet both were impaired (and differentially so) at deriving the global shape from multielement displays.

A rather different view on this matter has focused on relative spatial frequency. According to Ivry and Robertson (1998; Robertson \& Ivry, 2000), there is a secondary stage of processing that is sensitive to the relative rather than absolute spatial frequencies in the image, and this stage is functionally asymmetric and associated with more anterior cortical regions than those that register the absolute frequencies. According to this account, the difference between global and local information is a difference along a continuum of spatial frequency. With respect to the hemispheres, the claim is that the two hemispheres are biased toward different information along the same dimension of spatial frequency (Ivry \& Robertson, 1998; Lamb, Robertson, \& Knight, 1990; Robertson \& Ivry, 2000; Robertson \& Lamb, 1991), with the result that the right hemisphere is preferentially biased to process global information and the left hemisphere local information. Although our finding that the patients have normal spatial frequency thresholds is not incompatible with Ivry and Robertson's approach, within their perspective, there does not appear to be a clear way to accommodate the finding that the few- and many-element displays are processed differently by normal participants and by 1 of our patients (S.M.) given that the spatial frequency of the elements is relatively higher than that of the configuration for both displays. A potential further complication is that S.M., who has a clearly defined right-hemisphere lesion, is still able to derive the global form. In light of these findings, we suggest that the processing of global and local components is tied more to organizational processes than to differences along a continuum of spatial frequency and its relation to hemispheric biases.

This is not to say that the two hemispheres play equivalent roles in perceptual organization, as they apparently do not, but it suggests that the means whereby organization occurs is not primarily dependent on hemispheric-tuned spatial frequency filters. Although the neuroimaging studies have obtained bilateral activation in posterior cortex in many integration tasks (Gerlach et al., 2002), this does not necessarily imply that there is an equal contribution of both hemispheres to this process. As revealed by patient H.J.A., a lesion to the right hemisphere alone can impair the ability to derive closure (Boucart \& Humphreys, 1992a, 1992b). Moreover, the relatively greater contribution of the right hemisphere to perceptual organization is also observed in split-brain patients: Corballis, Fendrich, Shapley, and Gazzaniga (1999) showed that whereas both hemispheres seemed to be equally capable of perceiving illusory contours, amodal completion is more readily achieved by the right hemisphere.

In conclusion, we have examined the perceptual organization and object recognition abilities of 2 visual agnosic patients to shed light on the nature of these psychological processes, how they relate to one another, and the possible underlying neural substrates. Our findings indicate that perceptual organization is not a unitary phenomenon but rather a multiplicity of processes, some of which are simpler, operate earlier, and are instantiated in lower level areas of visual cortex, such as grouping by collinearity. In contrast, other processes are more complex, operate later, and rely on higher order visual areas, such as grouping by closure and shape formation. It is these latter processes that are critical for object recognition. The failure to exploit these more complex, configural processes, despite the preserved ability to do basic grouping, gives rise to a deficit in object recognition. The implication of these findings is that the ability to organize elements into visual units is necessary but not sufficient for object identification and recognition. To appreciate the identity of an object, one must necessarily apprehend the internal structure and its emergent global form.

\section{References}

Bacon, W. F., \& Egeth, H. E. (1991). Local processes in pre-attentive feature detection. Journal of Experimental Psychology: Human Perception and Performance, 17, 77-90.

Badcock, C. J., Whitworth, F. A., Badcock, D. R., \& Lovegrove, W. J. (1990). Low-frequency filtering and processing of local-global stimuli. Perception, 19, 617-629.

Behrmann, M. (in press). Neuropsychological approaches to perceptual organization: Evidence from visual agnosia. In G. Rhodes \& M. Peterson (Eds.), Analytic and holistic processes in the perception of faces, objects and scenes. New York: Oxford University Press.

Behrmann, M., \& Kimchi, R. (in press). Perceptual organization in visual agnosia. In R. Kimchi, M. Behrmann, \& C. Olson (Eds.), Perceptual organization in vision: Behavioral and neural perspectives. Mahwah, NJ: Erlbaum.

Behrmann, M., Nelson, J., \& Sekuler, E. (1998). Visual complexity in letter-by-letter reading: "Pure" alexia is not so pure. Neuropsychologia, 36, 1115-1132.

Beller, H. K. (1971). Priming: Effects of advance information on matching. Journal of Experimental Psychology, 87, 176-182.

Ben-Av, M. B., \& Sagi, D. (1995). Perceptual grouping by similarity and proximity: Experimental results can be predicted by intensity autocorrelations. Vision Research, 35, 853-866.

Boucart, M., \& Humphreys, G. W. (1992a). The computation of perceptual structure from collinearity and closure: Normality and pathology. $\mathrm{Neu}$ ropsychologia, 30, 527-546.

Boucart, M., \& Humphreys, G. W. (1992b). Global shape cannot be attended without object identification. Journal of Experimental Psychology: Human Perception and Performance, 18, 785-806.

Boucart, M., Humphreys, G. W., \& Lorenceau, J. (1995). Automatic access to object identity: Global information, not particular physical dimensions, is important. Journal of Experimental Psychology: Human Perception and Performance, 21, 584-601.

Brainard, D. H. (1997). The Psychophysics Toolbox. Spatial Vision, 10, 433-436.

Bullier, J., \& Nowak, L. G. (1995). Parallel versus serial processing: New vistas on the distributed organization of the visual system. Current Opinion in Neurobiology, 5, 497-503.

Butter, C. M., \& Trobe, J. D. (1994). Integrative agnosia following progressive multifocal leukoencephalopathy. Cortex, 30, 145-158.

Chainay, H., \& Humphreys, G. W. (2001). The real-object advantage in agnosia: Evidence for a role of surface and depth information in object recognition. Cognitive Neuropsychology, 18, 175-191.

Cohen, J. D., MacWhinney, B., Flatt, M., \& Provost, J. (1993). PsyScope: A new graphic interactive environment for designing psychology experiments. Behavioral Research Methods, Instruments and Computers, 25, $257-271$. 
Coltheart, M. (2002). Cognitive neuropsychology. In J. Wixted (Ed.), Steven's handbook of experimental psychology: Vol. 4. Methodology (3rd ed., pp. 139-174). New York: Wiley.

Corballis, P. M., Fendrich, R., Shapley, R. M., \& Gazzaniga, M. S. (1999). Illusory contour perception and amodal boundary completion: Evidence of a dissociation following callosotomy. Journal of Cognitive Neuroscience, 11, 459-466.

Davidoff, J., \& Warrington, E. K. (1999). The bare bones of object recognition: Implications from a case of object recognition impairment. Neuropsychologia, 37, 279-292.

Dell'Acqua, R., Job, R., \& Grainger, J. (2001). Is global shape sufficient for automatic object identification? Visual Cognition, 8, 801-822.

Doniger, G., Foxe, J. J., Murray, M. M., Higgins, B. A., Snodgrass, J. G., Schroeder, C. E., \& Javitt, D. C. (2000). Activation timecourse of ventral visual stream object-recognition areas: High density electrical mapping of perceptual closure processes. Journal of Cognitive Neuroscience, 12, 615-621.

Donnelly, N., Humphreys, G. W., \& Riddoch, M. J. (1991). Parallel computations of primitive shape descriptions. Journal of Experimental Psychology: Human Perception and Performance, 17, 561-570.

Driver, J., Davis, G., Russell, C., Turatto, M., \& Freeman, E. (2001). Segmentation, attention and phenomenal visual objects. Cognition, 80, $61-95$.

Efron, R. (1968). What is perception? Boston Studies in Philosophy of Science, 4, 137-173.

Enns, J. T., \& Kingstone, A. (1995). Access to global and local properties in visual search for compound stimuli. Psychological Science, 6, 283291.

Farah, M. J. (1990). Visual agnosia: Disorders of object recognition and what they tell us about normal vision. Cambridge, MA: MIT Press.

Feldman, J. (1999). The role of objects in perceptual grouping. Acta Psychologica, 102, 137-163.

Feldman, J. (2000). Bias toward regular form in mental shapes. Journal of Experimental Psychology: Human Perception and Performance, 26, 152-165.

ffytche, D. H., \& Zeki, S. (1996). Brain activity related to the perception of illusory contours. NeuroImage, 3, 104-108.

Fink, G. R., Halligan, P. W., Marshall, J. C., Frith, C. D., Frackowiak, R. S. J., \& Dolan, R. J. (1996, August 15). Where in the brain does visual attention select the forest and the trees? Nature, 382, 626-628.

Fink, G. R., Halligan, P. W., Marshall, J. C., Frith, C. D., Frackowiak, R. S. J., \& Dolan, R. J. (1997). Neural mechanisms involved in the processing of global and local aspects of hierarchically organized visual stimuli. Brain, 120, 1779-1791.

Gauthier, I., Behrmann, M., \& Tarr, M. J. (1999). Can face recognition really be dissociated from object recognition? Journal of Cognitive Neuroscience, 11, 349-370.

Gauthier, I., \& Tarr, M. J. (2002). Unraveling mechanisms for expert object recognition: Bridging brain activity and behavior. Journal of Experimental Psychology: Human Perception and Performance, 28, 431-446.

Georgopoulos, A. P., Wang, K., Georgopoulos, M. A., Tagaris, G. A., Amirikian, B., Richter, W., et al. (2001). Functional magnetic resonance imaging of visual object construction and shape discrimination: Relations among task, hemispheric lateralization, and gender. Journal of Cognitive Neuroscience, 13, 72-89.

Gerlach, C., Aaside, C. T., Humphreys, G. W., Gade, A., Paulson, O. B., $\&$ Law, I. (2002). Brain activity related to integrative processes in visual object recognition: Bottom-up integration and the modulatory influence of stored knowledge. Neuropsychologia, 40, 1254-1267.

Giersch, A., Humphreys, G., Boucart, M., \& Kovács, I. (2000). The computation of occluded contours in visual agnosia: Evidence for early computation prior to shape binding and figure-ground coding. Cognitive Neuropsychology, 17, 731-759.

Ginsburg, A. P. (1986). Spatial filtering and visual form information. In
K. R. Boff, L. Kaufman, \& J. P. Thomas (Eds.), Handbook of human perception and performance (pp. 1-41). New York: Wiley.

Goldmeier, E. (1972). Similarity in visually perceived forms. Psychological Issues, 8(Suppl. 1, Whole No. 29). (Original work published 1936)

Goodglass, H., Kaplan, E., \& Weintraub, S. (O. Segal, Illus.) (1983). Boston Naming Test (2nd ed.). Philadelphia: Lea \& Febiger.

Han, S., \& Humphreys, G. W. (1999). Interactions between perceptual organization based on Gestalt laws and those based on hierarchical processing. Perception \& Psychophysics, 61, 1287-1298.

Han, S., Humphreys, G. W., \& Chen, L. (1999a). Parallel and competitive processes in hierarchical analysis: Perceptual grouping and encoding of closure. Journal of Experimental Psychology: Human Perception and Performance, 25, 1411-1432.

Han, S., Humphreys, G. W., \& Chen, L. (1999b). Uniform connectedness and classical Gestalt principles of perceptual grouping. Perception \& Psychophysics, 61, 661-674.

Hasson, U., Hendler, T., Ben Bashat, D., \& Malach, R. (2001). Vase or face? A neural correlate of shape-selective grouping processes in the human brain. Journal of Cognitive Neuroscience, 13, 744-753.

Hughes, H. C., Fendrich, R., \& Reuter-Lorenz, P. (1990). Global versus local processing in the absence of low spatial frequencies. Journal of Cognitive Neuroscience, 2, 272-282.

Humphrey, G. K., Goodale, M. A., Jakobson, L. S., \& Servos, P. (1994). The role of surface information in object recognition: Studies of a visual form agnosic and normal subjects. Perception, 23, 1457-1481.

Humphreys, G. W. (1999). Integrative agnosia. In G. W. Humphreys (Ed.), Case studies in vision (pp. 41-58). London: Psychology Press.

Humphreys, G. W., \& Riddoch, M. J. (1987). To see but not to see: A case study of visual agnosia. Hillsdale, NJ: Erlbaum.

Humphreys, G. W., \& Riddoch, M. J. (2001). Neuropsychological disorders of visual object recognition and naming. In F. Boller \& J. Grafman (Series Eds.) \& M. Behrmann (Vol. Ed.), Handbook of neuropsychology: Vol. 4. Disorders of visual behavior (2nd ed., pp. 159-180). Amsterdam: Elsevier Science.

Humphreys, G. W., Riddoch, M. J., Donnelly, N., Freeman, T., Boucart, M., \& Muller, H. M. (1994). Intermediate visual processing and visual agnosia. In M. J. Farah \& G. Ratcliff (Eds.), The neuropsychology of high-level vision (pp. 63-101). Hillsdale, NJ: Erlbaum.

Humphreys, G. W., Riddoch, M. J., Quinlan, P. T., Price, C. J., \& Donnelly, N. (1992). Parallel pattern processing and visual agnosia. Canadian Journal of Psychology, 46, 377-416.

Ivry, R., \& Robertson, L. C. (1998). The two sides of perception. Cambridge, MA: MIT Press.

Jankowiak, J., Kinsbourne, M., Shalev, R. S., \& Bachman, D. L. (1992). Preserved visual imagery and categorization in a case of associative visual agnosia. Journal of Cognitive Neuroscience, 4, 119-131.

Kartsounis, L., \& Warrington, E. K. (1991). Failure of object recognition due to a breakdown in figure-ground discrimination in a patient with normal acuity. Neuropsychologia, 29, 969-980.

Kellman, P. J. (2000). An update on Gestalt psychology. In B. Landau, J. Sabini, E. Newport, \& J. Jonides (Eds.), Essays in honor of Henry and Lila Gleitman (pp. 157-190). Cambridge, MA: MIT Press.

Kellman, P. J., \& Shipley, T. F. (1991). A theory of visual interpolation in object perception. Cognitive Psychology, 23, 141-221.

Kimchi, R. (1988). Selective attention to global and local levels in the comparison of hierarchical patterns. Perception \& Psychophysics, 43, $189-198$.

Kimchi, R. (1992). Primacy of wholistic processing and global/local paradigm: A critical review. Psychological Bulletin, 112, 24-38.

Kimchi, R. (1994). The role of wholistic/configural properties versus global properties in visual form perception. Perception, 23, 489-504.

Kimchi, R. (1998). Uniform connectedness and grouping in the perceptual organization of hierarchical patterns. Journal of Experimental Psychology: Human Perception and Performance, 24, 1105-1118. 
Kimchi, R. (2000). The perceptual organization of visual objects: A microgenetic analysis. Vision Research, 40, 1333-1347.

Kimchi, R., \& Hadad, R. (2002). Influence of past experience on perceptual grouping. Psychological Science, 13, 41-47.

Kimchi, R., \& Palmer, S. (1982). Form and texture in hierarchically constructed patterns. Journal of Experimental Psychology: Human Perception and Performance, 8, 521-535.

Kimchi, R., \& Palmer, S. E. (1985). Separability and integrality of global and local levels of hierarchical patterns. Journal of Experimental Psychology: Human Perception and Performance, 11, 673-688.

Koehler, W. (1928). An aspect of Gestalt psychology. In C. Murchison (Ed.), Psychologies of 1925 (pp. 163-195). Worcester, MA: Clark University Press.

Koffka, K. (1935). Principles of Gestalt psychology. New York: Harcourt Brace Jovanovich.

Kohler, W. (1950). Physical gestalten. In W. D. Ellis (Ed.), A sourcebook of Gestalt psychology (pp. 17-54). New York: Humanities Press. (Original work published 1920)

Kovács, I., Kozma, P., Feher, A., \& Benedek, G. (1999). Late maturation of visual spatial integration in humans. Proceedings of the National Academy of Sciences, U.S.A., 96, 12204-12209.

Kovács, I., Polat, U., Pennefather, P. M., Chandna, A., \& Norcia, A. M. (2000). A new test of contour integration deficits in patients with a history of disrupted binocular experience during visual development. Vision Research, 40, 1775-1783.

Kurylo, D. D. (1997). Time course of perceptual grouping. Perception \& Psychophysics, 59, 142-147.

Lamb, M. R., \& Robertson, L. (1988). The processing of hierarchical stimuli: Effects of retinal locus, location uncertainty, and stimulus identity. Perception \& Psychophysics, 44, 172-181.

Lamb, M. R., Robertson, L. C., \& Knight, R. T. (1990). Component mechanisms underlying the processing of hierarchically organized patterns-Inferences from patients with unilateral cortical lesions. Journal of Experimental Psychology: Learning, Memory, and Cognition, 16, 471-483.

Lamb, M., \& Yund, E. W. (1993). The role of spatial frequency in the processing of hierarchically organized structure. Perception \& Psychophysics, 54, 773-784.

Lamme, V. A. F., \& Roelfsema, P. R. (2000). The distinct modes of vision offered by feedforward and recurrent processing. Trends in Neurosciences, $23,571-579$.

Lawson, R., \& Humphreys, G. W. (1999). The effects of view in depth on the identification of line drawings and silhouettes of familiar objects. Visual Cognition, 6, 165-195.

Lee, T. S. (in press). Computational and neural processes of attentive perceptual organization. In R. Kimchi, M. Behrmann, \& C. Olson (Eds.), Perceptual organization in vision: Behavioral and neural perspectives. Mahwah, NJ: Erlbaum.

Mack, A., Tang, B., Tuma, R., Kahn, S., \& Rock, I. (1992). Perceptual organization and attention. Cognitive Psychology, 24, 475-501.

Marotta, J., Behrmann, M., \& Genovese, C. (2001). A functional MRI study of face recognition in patients with prosopagnosia. NeuroReport, $12,1581-1582$.

Marotta, J. J., McKeeff, T., \& Behrmann, M. (2002). The effects of rotation and inversion on face processing in prosopagnosia. Cognitive Neuropsychology, 19, 31-47.

Marr, D. (1982). Vision. San Francisco: Freeman.

Marstrand, L., Gerlach, C., Udesen, H., \& Gade, A. (2000). Selective impairment of intermediate vision following stroke in the right occipital lobe. Journal of the International Neuropsychological Society, 6, 381.

Mendola, J., Dale, A. M., Fischl, B., Liu, A. K., \& Tootell, R. B. H. (1999). The representation of illusory and real contours in human cortical visual areas revealed by functional magnetic resonance imaging. Journal of Neuroscience, 19, 8560-8572.

Moore, C., \& Egeth, H. (1997). Perception without attention: Evidence of grouping under conditions of inattention. Journal of Experimental Psychology: Human Perception and Performance, 23, 339-352.

Mycroft, R., Behrmann, M., \& Kay, J. (2002). Letter-by-letter reading in visual agnosia. Manuscript in preparation.

Navon, D. (1977). Forest before trees: The precedence of global features in visual perception. Cognitive Psychology, 9, 353-383.

Neisser, U. (1967). Cognitive psychology. New York: Appleton Century Crofts.

Op de Beeck, H., Beatse, E., Wagemans, J., Sunaert, S., \& Van Hecke, P. (2000). The representation of shape in the context of visual object categorization tasks. NeuroImage, 12, 28-40.

Palmer, S. E. (2001). Understanding perceptual organization and grouping. In R. Kimchi, M. Behrmann, \& C. Olson (Eds.), Perceptual organization: Behavioral and neural processes. Hillsdale, NJ: Erlbaum.

Palmer, S., Neff, J., \& Beck, D. (1996). Late influences on perceptual grouping: Amodal completion. Psychonomic Bulletin \& Review, 3, 7580.

Palmer, S. E., \& Rock, I. (1994). Rethinking perceptual organization: The role of uniform connectedness. Psychonomic Bulletin \& Review, 1, $29-55$.

Paquet, L., \& Merikle, P. M. (1984). Global precedence: The effect of exposure duration. Canadian Journal of Psychology, 38, 45-53.

Pennefather, P. M., Chandna, A., Kovács, I., Polat, U., \& Norcia, A. M. (1999). Contour detection threshold: Repeatability and learning with "contour cards." Spatial Vision, 2, 257-266.

Peterson, M. A., \& Gibson, B. S. (1994a). Must shape recognition follow figure-ground organization? An assumption in peril. Psychological Science, 9, 253-259.

Peterson, M. A., \& Gibson, B. S. (1994b). Object recognition contributions to figure-ground organization: Operations on outlines and subjective contours. Perception \& Psychophysics, 56, 551-554.

Pomerantz, J. R. (1983). Global and local precedence: Selective attention in form and motion perception. Journal of Experimental Psychology: General, 112, 516-540.

Pomerantz, J. R., \& Pristach, E. A. (1989). Emergent features, attention, and perceptual glue in visual form perception. Journal of Experimental Psychology: Human Perception and Performance, 15, 635-649.

Ratcliff, G., \& Newcombe, F. A. (1982). Object recognition: Some deductions from the clinical evidence. In A. W. Ellis (Ed.), Normality and pathology in cognitive functions (pp. 147-171). New York: Academic Press.

Razpurker-Apfeld, I., \& Kimchi, R. (2003). Grouping: Time course and attentional demands. Manuscript in preparation.

Rensink, R., \& Enns, J. T. (1995). Preemption effects in visual search: Evidence for low-level grouping. Psychological Review, 102, 101-130.

Ricci, R., Vaishnavi, S., \& Chatterjee, A. (1999). A deficit of intermediate vision: Experimental observations and theoretical implications. Neurocase, 5, 1-12.

Riddoch, M. J., \& Humphreys, G. W. (1987). A case of integrative visual agnosia. Brain, 110, 1431-1462.

Riddoch, M. J., \& Humphreys, G. W. (1993). Birmingham Object Recognition Battery. Hove, England: Erlbaum.

Robertson, L. C., \& Ivry, R. (2000). Hemispheric asymmetries: Attention to visual and auditory primitives. Current Directions in Psychological Science, 9, 59-63.

Robertson, L. C., \& Lamb, M. R. (1991). Neuropsychological perspectives on theories of part/whole organization. Cognitive Psychology, 23, 299330.

Rock, I. (1986). The description and analysis of object and event perception. In K. R. Boff, L. Kaufman, \& J. P. Thomas (Eds.), Handbook of perception and human performance (Vol. 33, pp. 1-71). New York: Wiley.

Rock, I., \& Brosgole, L. (1964). Grouping based on phenomenal proximity. Journal of Experimental Psychology, 67, 531-538. 
Rock, I., Nijhawan, R., Palmer, S. E., \& Tudor, L. (1992). Grouping based on phenomenal similarity of achromatic color. Perception, 21, 779-789.

Sanocki, T., \& Sellers, E. (2001). Shifting resources to recognize a forming object: Dependencies involving object properties. Visual Cognition, 8, 197-235.

Sasaki, Y., Hadjikani, N., Fischl, B., Liu, A. K., Marret, S., Dale, A. M., \& Tootell, R. B. H. (2001). Local and global attention are mapped retinotopically in human occipital cortex. Proceedings of the National Academy of Sciences, U.S.A., 98, 2077-2082.

Sekuler, A. B., \& Palmer, S. E. (1992). Perception of partly occluded objects: A microgenetic analysis. Journal of Experimental Psychology: General, 121, 95-111.

Shipley, T. F., \& Kellman, P. (1992). Perception of occluded objects and illusory figures: Evidence for an identity hypothesis. Journal of Experimental Psychology: Human Perception and Performance, 18, 106-120.

Shulman, G. L., Sullivan, M. A., Gish, K., \& Sakoda, W. J. (1986). The role of spatial-frequency channels in the perception of local and global structure. Perception, 15, 259-273.

Shulman, G. L., \& Wilson, J. (1987). Spatial frequency and selective attention to local and global information. Neuropsychologia, 18, 89101.

Snodgrass, S. G., \& Vanderwart, M. A. (1980). A standardized set of 260 pictures: Norms for name agreement, image agreement, familiarity, and visual complexity. Journal of Experimental Psychology: Human Learning and Memory, 6, 174-215.

Sugita, Y. (1999, September 16). Grouping of image fragments in primary visual cortex. Nature, 401, 269-272.

Treisman, A. (1982). Perceptual grouping and attention in visual search for features and for objects. Journal of Experimental Psychology: Human Perception and Performance, 8, 194-214.

Treisman, A., Kahneman, D., \& Burkell, J. (1983). Perceptual objects and the cost of filtering. Perception \& Psychophysics, 33, 527-532.
Trick, L. M., \& Enns, J. M. (1997). Clusters precede shapes in perceptual organization. Psychological Science, 8, 124-129.

Vecera, S. P., \& O'Reilly, R. (1998). Figure-ground organization and object recognition processes: An interactive account. Journal of Experimental Psychology: Human Perception and Performance, 24, 441-462.

Vecera, S. P., \& O'Reilly, R. C. (2000). Graded effects in hierarchical figure-ground organization: Reply to Peterson (1999). Journal of Experimental Psychology: Human Perception and Performance, 26, 12211231.

von der Heydt, R., \& Peterhans, E. (1989). Mechanisms of contour perception in monkey visual cortex. Journal of Neuroscience, 9, 17311748.

von der Heydt, R., Peterhans, E., \& Baumgartner, G. (1984, June 15). Illusory contours and cortical neuron responses. Science, 224, $1260-$ 1262.

Wertheimer, M. (1950). Untersuchungen zur Lehre von der Gestalt, II [Laws of organization in perceptual forms]. Psychologische Forschung, 4, 301-350. (Original work published 1923)

Westheimer, G. (1999). Gestalt theory reconfigured: Max Wertheimer's anticipation of recent developments in visual neuroscience. Perception, $18,5-15$.

Yovel, G., Yovel, I., \& Levy, J. (2001). Hemispheric asymmetries for global and local visual perception: Effects of stimulus and task factors. Journal of Experimental Psychology: Human Perception and Performance, 27, 1369-1385.

Zhou, H., Friedman, H. S., \& von der Heydt, R. (2000). Coding of border ownership in monkey visual cortex. Journal of Neuroscience, 20, $6594-$ 6611.

Received July 3, 2001

Revision received January 18, 2002

Accepted May 16, 2002 